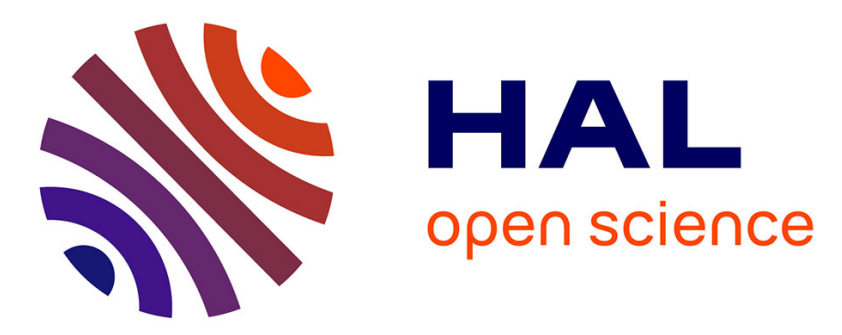

\title{
A procedure to find equivalences among dynamic models of planar biped robots
}

Víctor Santibañez, Javier Moreno-Valenzuela, Victor de Leon Gomez

\section{To cite this version:}

Víctor Santibañez, Javier Moreno-Valenzuela, Victor de Leon Gomez. A procedure to find equivalences among dynamic models of planar biped robots. Simulation Modelling Practice and Theory, 2017. hal01728357

\section{HAL Id: hal-01728357 \\ https://hal.science/hal-01728357}

Submitted on 10 Mar 2018

HAL is a multi-disciplinary open access archive for the deposit and dissemination of scientific research documents, whether they are published or not. The documents may come from teaching and research institutions in France or abroad, or from public or private research centers.
L'archive ouverte pluridisciplinaire HAL, est destinée au dépôt et à la diffusion de documents scientifiques de niveau recherche, publiés ou non, émanant des établissements d'enseignement et de recherche français ou étrangers, des laboratoires publics ou privés. 


\title{
A procedure to find equivalences among dynamic models of planar biped robots
}

\author{
Víctor De-León-Gómez ${ }^{\mathrm{a}}$, Víctor Santibañez ${ }^{\mathrm{a}, *}$, Javier Moreno-Valenzuela $^{\mathrm{b}}$ \\ ${ }^{a}$ Tecnológico Nacional de México/Instituto Tecnológico de la Laguna. \\ Boulevard Revolución y Cuauhtémoc s/n, Torreón, Coahuila, 27000, México. \\ e-mail:vsdeleon@hotmail.com,santibanez@ieee.org \\ ${ }^{b}$ Instituto Politécnico Nacional-CITEDI. \\ Ave. Instituto Politécnico Nacional 1310, Tijuana, BC, 22435, México. \\ e-mail: moreno@citedi.mx
}

\begin{abstract}
In this paper, a method to find equivalences among dynamic models is presented. The models are given in different generalized coordinates for the same mechanical system. This novel method is valid for planar biped robots, i.e., those whose motions are executed only in a plane. We show that no matter which generalized coordinates are used to get the dynamic models, there is always an equivalence among them by using a particular input matrix. Without loss of generality, we exhibit some advantages of getting the dynamic model using absolute coordinates instead of relative coordinates, and we show how to calculate the input matrix for getting the equivalence between these models. Because of its simplicity, a compass-like biped robot model is used as example to explain in detail the novel procedure. In order to appreciate the benefits of the proposed procedure in biped robots of high degrees of freedom, we also present the equivalence between two dynamic models for the single support phase of a 5 degrees of freedom biped robot using absolute coordinates and relative ones.
\end{abstract}

\footnotetext{
* Corresponding author

Email address: santibanez@ieee.org, vsantiba@itlalaguna.edu.mx (Víctor Santibañez)
}

Preprint submitted to Simulation Modelling Practice and Theory 
Keywords: Dynamic model, dynamic equivalences , Lagrange's equations, biped robots, numerical simulations.

\section{Introduction}

The dynamic model of biped robots is divided into three main phases: the single support phase (SSP), where the robot is supported by one foot; the double support phase (DSP), where the system is supported by two feet; and the flying 5 phase $(\mathrm{FP})$, where no feet are touching the ground. This dynamic model is important in the development of control strategies and analysis of these robots, such as: the study of passive gaits without energy input [1, 2]; the design of energy based controllers that exploit passive features [3, 4]; the development of control techniques that attempt to achieve certain objectives in terms of motion velocity or energy consumption needed to produce a gait [5, 6, 7, 8, ; as well as stability analysis of the limit cycle of the closed-loop systems. In this paper, we will focus into the study of the SSP dynamic model, which can be obtained from the application of the Euler-Lagrange equation of motion and is similar to the model of planar manipulator robots.

The selection of the reference frame where each generalized coordinate will be measured to obtain the dynamic model is important. The reason is that the applied torques in the Euler-Lagrange equations could not include the dynamics of subsequent links. For many robotic systems, the generalized coordinates chosen to obtain the dynamic model are those measured from the extension of the previous link (relative generalized coordinates), except by the first one which is measured with respect to an inertial reference frame. We show that by using these relative generalized coordinates, the torque applied to each joint is directly obtained. On the other hand, not much has been said in the literature if the model is derived by selecting generalized coordinates measured

25 from different reference frame orientations. In this paper, we are focused on discussing such a problem. We show how to get different representations of dynamic models for the same system (representations with 
different elements in the inertia matrix, Coriolis matrix and gravitational vector) derived from the Euler-Lagrange equation of motion, tion planning. Although these dynamic models may look different, we prove that there exists a relationship among them. An example of a dynamic model representation is the one obtained by using absolute coordinates $\theta$ (instead of using relative generalized coordinates $q$ ) for coordinates $\theta$ has the following advantages:

- The elements into the matrices of a dynamic model obtained from Euler-Lagrange equation of motion with absolute coordinates $\theta$, are simpler than those obtained from using relative generalized coordinates $q$. Thus, the implementation of a dynamic model obtained by using the coordinate $\theta$, will help saving time of calculations in limited systems (such as embedded systems or in systems where optimization algorithms based on the dynamic model are used, etc.).

- The calculation of singularities [9] in the Jacobian matrix is easier by using absolute coordinates than the calculation of that obtained by using relative coordinates, since the determinant of the matrix is easier to compute.

- As known in literature, for biped robots it is particularly important the change of support at the impact of the free foot with the ground. So, by using absolute generalized coordinates $\theta$, the interchange of the position variables, among the stance and free legs, is straightforward.

- Also, by using $\theta$ will allow achieving a better walking analysis of the robot. The reason is that it allows analysing limit cycles of each joint by observing when that joint is part of the support leg or the free one, without having discontinuities in position. 
- It is easier to analyse the motion of a particular link by using absolute coordinates than relative ones, as will be shown in the simulations section of this paper.

On the other hand, some control design procedures may need specific matrix structures, for instance, they could need that inertia matrix $M$ depends only on the underactuated coordinate or the actuated one (for example in [10]). By choosing different generalized coordinates we could make $M$ accomplishes such a requisite. Nevertheless, there are a lot of possibilities for choosing generalized coordinates: some of them could be relatives, absolutes, measured from the positive vertical axis or measured from the negative one, measured from the extreme of a link, positive in clockwise or in counter-clockwise direction, etc. Thus, the resulting matrices would be different and would be useful for some specific purposes. The given analysis will also be helpful to understand how to calculate the nontrivial input matrix which appears multiplying the vector of applied torques in biped robot models reported in the literature, such as [11, 12, 13, 14, among others. This work also com75 plements those studies of the direct and inverse kinematics of planar redundant robots [15. In order to make clear the proposed procedure, we analyse in detail two models in SSP of the compass-like biped robot (CBR). Also we compare the dynamic model of a 5 degrees-of-freedom (DOF) biped robot obtained in two different ways, each one taking into account generalized coordinates for the robot link position measured from different reference frames. Thus, an input matrix is needed to make the equivalence between the two models.

Given the advantages of modelling the dynamics of planar biped R1C1 robots by using absolute generalized coordinates $\theta$, it arises the need of relating such a model with one obtained with relative generalized coordinates, whose elements in general are more complex, but they consider the whole dynamics of the robot including the reaction forces and torques of subsequent links, which are not considered in the absolute coordinate-based model because the fixed references frames 
decouple the links. In this paper, our main contribution is the introduction of a novel procedure to find equivalences among dynamic models of planar biped robots, making easy the whole modelling procedure. The benefits of our proposal are more evident in planar biped robots with high DOF.

This paper is organized as follows: in Section 2, the dynamic model of planar biped robots obtained from different reference frames is shown. In Section 3, the procedure to find the equivalence among dynamic models of the same planar biped robot is explained. In order to exemplify our approach, the CBR is analysed in Section 4. First, its dynamic model is obtained by taking into account the generalized coordinates measured from previous links (relatives coordinates, as is done with serial manipulator robots), and by taking into account the generalized coordinates measured from inertial frames (absolutes coordinates, as is normally used in the literature of biped robots, i.e., measured at the end of the feet from vertical axes). Simulations results are given in order to explain the difference between the torques obtained from the two dynamic models presented. The equivalence between the two obtained models is also shown. As another example, in Section 5, the equivalence between two dynamic models of a 5 DOF biped robot is presented. Here, relative and absolute coordinates are used to show the advantages of the proposed approach of equivalency between models. Finally the conclusions derived from our study are given in Section 6 .

\section{Dynamic models for planar biped robots in SSP}

The dynamic model of a planar biped robot in SSP can be obtained by applying the Euler-Lagrange equation of motion using generalized coordinates that define the robot configuration. It should be noticed that if different reference frames to measure the generalized coordinates are used, different dynamic model representations are obtained.

Usually, the generalized coordinates chosen for getting the dynamic model of a serial robot are those measured from the extension of the previous link, as 


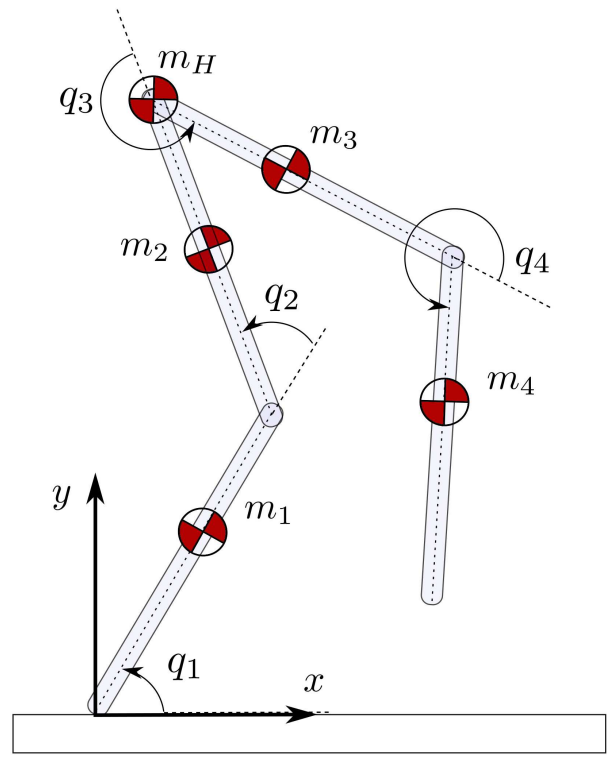

Figure 1: Kinematic model of a planar biped robot with relative generalized coordinates $\boldsymbol{q}$.

shown in Figure 1. Thus, by applying the Euler-Lagrange equation of motion, the dynamic model for this robot could be represented in a compact form as

$$
M(\boldsymbol{q}) \ddot{\boldsymbol{q}}+\boldsymbol{d}(\boldsymbol{q}, \dot{\boldsymbol{q}})=\boldsymbol{\tau}
$$

120 respectively, $M(\boldsymbol{q})=M(\boldsymbol{q})^{T}>0$ is the inertia matrix, $\boldsymbol{d}(\boldsymbol{q}, \dot{\boldsymbol{q}})$ is a vector with the remaining dynamic terms, and $\tau \in \mathbb{R}^{n}$ is the vector of applied torques. Each torque $\tau_{i}$ applied in each joint includes the whole dynamic of the robot due to the motion and masses of subsequent links.

On the other hand, without loss of generality, by choosing as generalized coordinates those measured from the horizonta 11 as shown in Figure 2 , and by applying the Euler-Lagrange equation of motion for getting the dynamic model,

\footnotetext{
${ }^{1}$ We can choose any other reference frame to measure the generalized coordinates, such as those measured from the vertical or from other links
} 


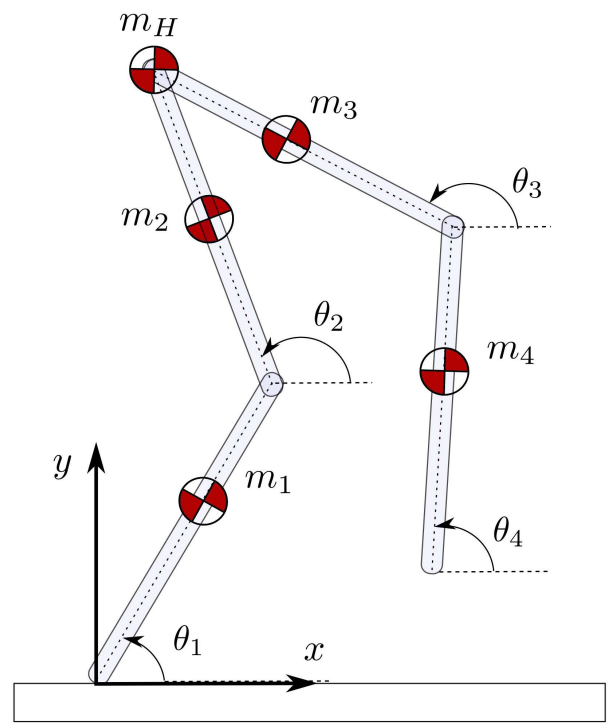

Figure 2: Kinematic model of a planar biped robot with absolute generalized coordinates $\boldsymbol{\theta}$.

we get

$$
\bar{M}(\boldsymbol{\theta}) \ddot{\boldsymbol{\theta}}+\overline{\boldsymbol{d}}(\boldsymbol{\theta}, \dot{\boldsymbol{\theta}})=\overline{\boldsymbol{\tau}}
$$

where $\boldsymbol{\theta}, \dot{\boldsymbol{\theta}}, \ddot{\boldsymbol{\theta}} \in \mathbb{R}^{n}$, are the vector of joint position, velocity and acceleration, respectively, $\bar{M}(\boldsymbol{\theta})=\bar{M}(\boldsymbol{\theta})^{T}>0$ is the inertia matrix, $\overline{\boldsymbol{d}}(\boldsymbol{\theta}, \dot{\boldsymbol{\theta}})$ is a vector with the rest of the dynamic terms, and $\bar{\tau} \in \mathbb{R}^{n}$ is the vector of quasi-applied torques. In this framework, the resulting inertia and Coriolis matrices are commonly simpler than those in equation (1). Nevertheless, the torque vector that appears in equation (2) does not include the whole dynamics of the system, such as reaction torques of subsequent links. In other words, the signal $\overline{\boldsymbol{\tau}}(t)$ can not be used as the applied torques at the joints for simulations or experimental purposes. However, it is possible to relate the simpler representation given by $\overline{\boldsymbol{\tau}}(t)$ in (2) to the applied torques at the joints $\boldsymbol{\tau}(t)$ given in (1), which considers the whole dynamic of the robot, as will be seen in the next. 
3. Equivalence among dynamic models obtained with different coordinates

A mechanical system expressed with generalized coordinates $\boldsymbol{q}$ has associated a Lagrangian given by

$$
L(\boldsymbol{q}, \dot{\boldsymbol{q}})=\mathcal{K}(\boldsymbol{q}, \dot{\boldsymbol{q}})-\mathcal{U}(\boldsymbol{q})
$$

where

$$
\mathcal{K}(\boldsymbol{q}, \dot{\boldsymbol{q}})=\frac{1}{2} \dot{\boldsymbol{q}}^{T} M(\boldsymbol{q}) \dot{\boldsymbol{q}}
$$

145 is the kinetic energy of the system and $\mathcal{U}(\boldsymbol{q})$ its potential energy.

By following the Euler-Lagrange equation of motion given by

$$
\frac{d}{d t}\left[\frac{\partial}{\partial \dot{\boldsymbol{q}}} L(\boldsymbol{q}, \dot{\boldsymbol{q}})\right]-\frac{\partial}{\partial \boldsymbol{q}} L(\boldsymbol{q}, \dot{\boldsymbol{q}})=\boldsymbol{\tau}
$$

the dynamic model of a mechanical system is obtained. Then, it is posible to achieve a simple representation of equation (5) as follows [16]:

$$
\frac{d}{d t}\left[\frac{\partial}{\partial \dot{\boldsymbol{q}}} \mathcal{K}(\boldsymbol{q}, \dot{\boldsymbol{q}})\right]-\frac{\partial}{\partial \boldsymbol{q}} \mathcal{K}(\boldsymbol{q}, \dot{\boldsymbol{q}})+\frac{\partial}{\partial \boldsymbol{q}} \mathcal{U}(\boldsymbol{q})=\boldsymbol{\tau},
$$

which can be also expressed by using (4) as

$$
\frac{d}{d t}\left[\frac{\partial}{\partial \dot{\boldsymbol{q}}}\left(\frac{1}{2} \dot{\boldsymbol{q}}^{T} M(\boldsymbol{q}) \dot{\boldsymbol{q}}\right)\right]-\frac{\partial}{\partial \boldsymbol{q}}\left(\frac{1}{2} \dot{\boldsymbol{q}}^{T} M(\boldsymbol{q}) \dot{\boldsymbol{q}}\right)+\frac{\partial}{\partial \boldsymbol{q}} \mathcal{U}(\boldsymbol{q})=\boldsymbol{\tau}
$$

150 where

$$
\begin{gathered}
\frac{\partial}{\partial \dot{\boldsymbol{q}}}\left(\frac{1}{2} \dot{\boldsymbol{q}}^{T} M(\boldsymbol{q}) \dot{\boldsymbol{q}}\right)=M(\boldsymbol{q}) \dot{\boldsymbol{q}} \\
\frac{d}{d t}\left[\frac{\partial}{\partial \dot{\boldsymbol{q}}}\left(\frac{1}{2} \dot{\boldsymbol{q}}^{T} M(\boldsymbol{q}) \dot{\boldsymbol{q}}\right)\right]=M(\boldsymbol{q}) \ddot{\boldsymbol{q}}+\dot{M}(\boldsymbol{q}) \dot{\boldsymbol{q}}
\end{gathered}
$$

and

$$
\frac{\partial}{\partial \boldsymbol{q}} \mathcal{U}(\boldsymbol{q})=\boldsymbol{g}(\boldsymbol{q})
$$

By using equations (7) and (8) into (6) we obtain a compact set of equations given by

$$
M(\boldsymbol{q}) \ddot{\boldsymbol{q}}+C(\boldsymbol{q}, \dot{\boldsymbol{q}}) \dot{\boldsymbol{q}}+\boldsymbol{g}(\boldsymbol{q})=\boldsymbol{\tau}
$$


with

$$
C(\boldsymbol{q}, \dot{\boldsymbol{q}}) \dot{\boldsymbol{q}}=\dot{M}(\boldsymbol{q}) \dot{\boldsymbol{q}}-\frac{1}{2} \frac{\partial}{\partial \boldsymbol{q}}\left(\dot{\boldsymbol{q}}^{T} M(\boldsymbol{q}) \dot{\boldsymbol{q}}\right)
$$

where $C(\boldsymbol{q}, \dot{\boldsymbol{q}})$ is the $n \times n$ centrifugal and Coriolis matrix and $\boldsymbol{g}(\boldsymbol{q})$ is the vector of gravitational forces and torques of dimension $n$.

The generalized coordinates $\boldsymbol{q}$ have a linear relation with other generalized coordinates $\boldsymbol{\theta}$, which is given by

$$
\boldsymbol{q}=A \boldsymbol{\theta}+\boldsymbol{b}
$$

being $A \in \mathbb{R}^{n \times n}$ a non singular constant relation matrix between $\boldsymbol{q}$ and $\boldsymbol{\theta}$, and $\boldsymbol{b} \in \mathbb{R}^{n}$ a constant vector. This relation is written as $\boldsymbol{q}=\boldsymbol{q}(\boldsymbol{\theta})$, such that $\dot{\boldsymbol{q}}=\dot{\boldsymbol{q}}(\dot{\boldsymbol{\theta}})=A \dot{\boldsymbol{\theta}}$ and $\ddot{\boldsymbol{q}}=\ddot{\boldsymbol{q}}(\ddot{\boldsymbol{\theta}})=A \ddot{\boldsymbol{\theta}}$. By applying these relations to the Lagrangian in equation $(3)$, we get

$$
L(\boldsymbol{q}(\boldsymbol{\theta}), \dot{\boldsymbol{q}}(\dot{\boldsymbol{\theta}}))=\mathcal{K}(\boldsymbol{q}(\boldsymbol{\theta}), \dot{\boldsymbol{q}}(\dot{\boldsymbol{\theta}}))-\mathcal{U}(\boldsymbol{q}(\boldsymbol{\theta}))
$$

Therefore, the computing of the Euler-Lagrange equation of motion for the new generalized coordinates $\boldsymbol{\theta}$ is given by

$$
\frac{d}{d t}\left[\frac{\partial}{\partial \dot{\boldsymbol{\theta}}} L(\boldsymbol{q}(\boldsymbol{\theta}), \dot{\boldsymbol{q}}(\dot{\boldsymbol{\theta}}))\right]-\frac{\partial}{\partial \boldsymbol{\theta}} L(\boldsymbol{q}(\boldsymbol{\theta}), \dot{\boldsymbol{q}}(\dot{\boldsymbol{\theta}}))=\overline{\boldsymbol{\tau}}
$$

which can also be written as

$$
\frac{d}{d t}\left[\frac{\partial}{\partial \dot{\boldsymbol{\theta}}} \mathcal{K}(\boldsymbol{q}(\boldsymbol{\theta}), \dot{\boldsymbol{q}}(\dot{\boldsymbol{\theta}}))\right]-\frac{\partial}{\partial \boldsymbol{\theta}} \mathcal{K}(\boldsymbol{q}(\boldsymbol{\theta}), \dot{\boldsymbol{q}}(\dot{\boldsymbol{\theta}}))+\frac{\partial}{\partial \boldsymbol{\theta}} \mathcal{U}(\boldsymbol{q}(\boldsymbol{\theta}))=\overline{\boldsymbol{\tau}}
$$

Note that, the generalized forces $\bar{\tau}$ for equation 11 is not equivalent to $\tau$ in equation (5) since Euler-Lagrange equations are being computed with respect to coordinate $\boldsymbol{\theta}$. However, there is a relationship between $\overline{\boldsymbol{\tau}}$ and $\boldsymbol{\tau}$ that we will show in the next. By using the chain rule for the first term of the left hand side of 11 we get

$$
\begin{aligned}
\frac{\partial}{\partial \dot{\boldsymbol{\theta}}} \mathcal{K}(\boldsymbol{q}(\boldsymbol{\theta}), \dot{\boldsymbol{q}}(\dot{\boldsymbol{\theta}})) & =\left[\frac{\partial \mathcal{K}^{T}}{\partial \boldsymbol{q}} \frac{\partial \boldsymbol{q}}{\partial \dot{\boldsymbol{\theta}}}\right]^{T}+\left[\frac{\partial \mathcal{K}^{T}}{\partial \dot{\boldsymbol{q}}} \frac{\partial \dot{\boldsymbol{q}}}{\partial \dot{\boldsymbol{\theta}}}\right]^{T} \\
& =0+\left[[M(\boldsymbol{q}(\boldsymbol{\theta})) \dot{\boldsymbol{q}}(\dot{\boldsymbol{\theta}})]^{T} A\right]^{T} \\
& =A^{T} M(\boldsymbol{q}(\boldsymbol{\theta})) \dot{\boldsymbol{q}}(\dot{\boldsymbol{\theta}}),
\end{aligned}
$$


where $\frac{\partial \dot{\boldsymbol{q}}}{\partial \dot{\boldsymbol{\theta}}}=A$, and the time derivative of 12 is given by

$$
\begin{aligned}
\frac{d}{d t}\left[\frac{\partial}{\partial \dot{\boldsymbol{\theta}}} \mathcal{K}(\boldsymbol{q}(\boldsymbol{\theta}), \dot{\boldsymbol{q}}(\dot{\boldsymbol{\theta}}))\right]=\quad & A^{T} M(\boldsymbol{q}(\boldsymbol{\theta})) \ddot{\boldsymbol{q}}(\ddot{\boldsymbol{\theta}}) \\
& +A^{T} \dot{M}(\boldsymbol{q}(\boldsymbol{\theta})) \dot{\boldsymbol{q}}(\dot{\boldsymbol{\theta}}) .
\end{aligned}
$$

By using again the chain rule for the second term of the left hand side of (11) we have

$$
\begin{aligned}
\frac{\partial}{\partial \boldsymbol{\theta}} \mathcal{K}(\boldsymbol{q}(\boldsymbol{\theta}), \dot{\boldsymbol{q}}(\dot{\boldsymbol{\theta}})) & =\left[\frac{\partial \mathcal{K}^{T}}{\partial \boldsymbol{q}} \frac{\partial \boldsymbol{q}}{\partial \boldsymbol{\theta}}\right]^{T}+\left[\frac{\partial \mathcal{K}^{T}}{\partial \dot{\boldsymbol{q}}} \frac{\partial \dot{\boldsymbol{q}}}{\partial \boldsymbol{\theta}}\right]^{T} \\
& =\left[\left[\frac{1}{2} \frac{\partial}{\partial \boldsymbol{q}}\left(\dot{\boldsymbol{q}}(\dot{\boldsymbol{\theta}})^{T} M(\boldsymbol{q}(\boldsymbol{\theta})) \dot{\boldsymbol{q}}(\dot{\boldsymbol{\theta}})\right)\right]^{T} A\right]^{T}+0 \\
& =\frac{1}{2} A^{T} \frac{\partial}{\partial \boldsymbol{q}}\left(\dot{\boldsymbol{q}}(\dot{\boldsymbol{\theta}})^{T} M(\boldsymbol{q}(\boldsymbol{\theta})) \dot{\boldsymbol{q}}(\dot{\boldsymbol{\theta}})\right)
\end{aligned}
$$

175 where $\frac{\partial \boldsymbol{q}}{\partial \boldsymbol{\theta}}=A$. By doing the same for the third term of the left hand side of (11) we get

$$
\begin{aligned}
\frac{\partial}{\partial \boldsymbol{\theta}} \mathcal{U}(\boldsymbol{q}(\boldsymbol{\theta})) & =\left[{\frac{\partial \mathcal{U}(\boldsymbol{q}(\boldsymbol{\theta}))^{T}}{\partial \boldsymbol{q}}}^{T} \frac{\partial \boldsymbol{q}}{\partial \boldsymbol{\theta}}\right]^{T} \\
& =\left[\frac{\partial \mathcal{U}(\boldsymbol{q}(\boldsymbol{\theta}))^{T}}{\partial \boldsymbol{q}} A\right]^{T} \\
& =A^{T} \frac{\partial \mathcal{U}(\boldsymbol{q}(\boldsymbol{\theta}))}{\partial \boldsymbol{q}}
\end{aligned}
$$

Then, by substituting equations (13)- 1 (15) into equation (11), we obtain

$$
\begin{aligned}
& A^{T} M(\boldsymbol{q}(\boldsymbol{\theta})) \ddot{\boldsymbol{q}}(\ddot{\boldsymbol{\theta}})+A^{T} \dot{M}(\boldsymbol{q}(\boldsymbol{\theta})) \dot{\boldsymbol{q}}(\dot{\boldsymbol{\theta}}) \\
& -\frac{1}{2} A^{T} \frac{\partial}{\partial \boldsymbol{q}}\left(\dot{\boldsymbol{q}}(\dot{\boldsymbol{\theta}})^{T} M(\boldsymbol{q}(\boldsymbol{\theta})) \dot{\boldsymbol{q}}(\dot{\boldsymbol{\theta}})\right)+A^{T} \frac{\partial \mathcal{U}(\boldsymbol{q}(\boldsymbol{\theta}))}{\partial \boldsymbol{q}}=\overline{\boldsymbol{\tau}},
\end{aligned}
$$

where, according to equations $(10)$ and $(8)$, we can write

$$
A^{T} C(\boldsymbol{q}(\boldsymbol{\theta}), \dot{\boldsymbol{q}}(\dot{\boldsymbol{\theta}})) \dot{\boldsymbol{q}}(\dot{\boldsymbol{\theta}})=A^{T}\left[\dot{M}(\boldsymbol{q}(\boldsymbol{\theta})) \dot{\boldsymbol{q}}(\dot{\boldsymbol{\theta}})-\frac{1}{2} \frac{\partial}{\partial \boldsymbol{q}}\left(\dot{\boldsymbol{q}}(\dot{\boldsymbol{\theta}})^{T} M(\boldsymbol{q}(\boldsymbol{\theta})) \dot{\boldsymbol{q}}(\dot{\boldsymbol{\theta}})\right)\right],
$$

and

$$
A^{T} \boldsymbol{g}(\boldsymbol{q}(\boldsymbol{\theta}))=A^{T} \frac{\partial \mathcal{U}(\boldsymbol{q}(\boldsymbol{\theta}))}{\partial \boldsymbol{q}}
$$


so that equation 16 can be expressed as

$$
A^{T} M(\boldsymbol{q}(\boldsymbol{\theta})) \ddot{\boldsymbol{q}}(\ddot{\boldsymbol{\theta}})+A^{T} C(\boldsymbol{q}(\boldsymbol{\theta}), \dot{\boldsymbol{q}}(\dot{\boldsymbol{\theta}})) \dot{\boldsymbol{q}}(\dot{\boldsymbol{\theta}})+A^{T} \boldsymbol{g}(\boldsymbol{q}(\boldsymbol{\theta}))=\overline{\boldsymbol{\tau}}
$$

Finally, by replacing the relations $\boldsymbol{q}=A \boldsymbol{\theta}+\boldsymbol{b}, \dot{\boldsymbol{q}}=A \dot{\boldsymbol{\theta}}$ and $\ddot{\boldsymbol{q}}=A \ddot{\boldsymbol{\theta}}$, we obtain

$$
A^{T} M(A \boldsymbol{\theta}+\boldsymbol{b}) A \ddot{\boldsymbol{\theta}}+A^{T} C(A \boldsymbol{\theta}+\boldsymbol{b}, A \dot{\boldsymbol{\theta}}) A \dot{\boldsymbol{\theta}}+A^{T} \boldsymbol{g}(A \boldsymbol{\theta}+\boldsymbol{b})=\overline{\boldsymbol{\tau}},
$$

which in compact form is given by

$$
\bar{M}(\boldsymbol{\theta}) \ddot{\boldsymbol{\theta}}+\bar{C}(\boldsymbol{\theta}, \dot{\boldsymbol{\theta}}) \dot{\boldsymbol{\theta}}+\overline{\boldsymbol{g}}(\boldsymbol{\theta})=\overline{\boldsymbol{\tau}}
$$

where

$$
\begin{aligned}
\bar{M}(\boldsymbol{\theta}) & =A^{T} M(A \boldsymbol{\theta}+\boldsymbol{b}) A \\
\bar{C}(\boldsymbol{\theta}, \dot{\boldsymbol{\theta}}) & =A^{T} C(A \boldsymbol{\theta}+\boldsymbol{b}, A \dot{\boldsymbol{\theta}}) A, \\
\overline{\boldsymbol{g}}(\boldsymbol{\theta}) & =A^{T} \boldsymbol{g}(A \boldsymbol{\theta}+\boldsymbol{b}) .
\end{aligned}
$$

Moreover, equation (17) can be rearranged so that

$$
A^{T}[M(\boldsymbol{q}) \ddot{\boldsymbol{q}}+C(\boldsymbol{q}, \dot{\boldsymbol{q}}) \dot{\boldsymbol{q}}+\boldsymbol{g}(\boldsymbol{q})]=\overline{\boldsymbol{\tau}},
$$

185

where dependencies on $\boldsymbol{\theta}, \dot{\boldsymbol{\theta}}$ and $\ddot{\boldsymbol{\theta}}$ were omitted. Now, by substituting equation (9) into equation 22 we get

$$
A^{T} \boldsymbol{\tau}=\bar{\tau}
$$

which is the relationship between the torques of both models. Thus, we can use the simplest dynamic model representation, commonly obtained from the left hand side of equation (18), combined with the torques that consider the whole dynamic obtained from right hand side of equation $(9)$ as follows:

$$
\bar{M}(\boldsymbol{\theta}) \ddot{\boldsymbol{\theta}}+\bar{C}(\boldsymbol{\theta}, \dot{\boldsymbol{\theta}}) \dot{\boldsymbol{\theta}}+\overline{\boldsymbol{g}}(\boldsymbol{\theta})=A^{T} \boldsymbol{\tau}
$$

Notice that, from the fact of the relation matrix $A$ is nonsingular, R1C4 this procedure can also be used to find $M(\boldsymbol{q}), C(\boldsymbol{q}, \dot{\boldsymbol{q}})$ and $\boldsymbol{g}(\boldsymbol{q})$ starting 
from $\bar{M}(\boldsymbol{\theta}), \bar{C}(\boldsymbol{\theta}, \dot{\boldsymbol{\theta}})$ and $\overline{\boldsymbol{g}}(\boldsymbol{\theta})$, by means of the following equivalence equations obtained from (19), 20), 21):

$$
\begin{aligned}
M(\boldsymbol{q}) & =A^{-T} \bar{M}\left(A^{-1} \boldsymbol{q}-A^{-1} \boldsymbol{b}\right) A^{-1}, \\
C(\boldsymbol{q}, \dot{\boldsymbol{q}}) & =A^{-T} \bar{C}\left(A^{-1} \boldsymbol{q}-A^{-1} \boldsymbol{b}, A^{-1} \dot{\boldsymbol{q}}\right) A^{-1}, \\
\boldsymbol{g}(\boldsymbol{q}) & =A^{-T} \overline{\boldsymbol{g}}\left(A^{-1} \boldsymbol{q}-A^{-1} \boldsymbol{b}\right) .
\end{aligned}
$$

As an additional fact, since the total energy $E$ of a mechanical system is conserved (if dissipative forces as friction are not taken into account), we can note that, regardless of which generalized coordinate is used to represent the system, the power obtained from each one of these system representations is the same, i.e.,

$$
\dot{E}=\dot{\boldsymbol{q}}^{T} \boldsymbol{\tau}=\dot{\boldsymbol{\theta}}^{T} \overline{\boldsymbol{\tau}}
$$

where $\dot{E}$ is the power consumption of the system (see proof in Appendix).

\section{The compass-like biped robot}

In order to show the advantages of the proposed method of equivalence between models, we present a discussion for the CBR. Specifically, the relative coordinate-based model and the absolute coordinate-based are developed and simulations in order to show the relevance of the generalized forces in both models are given. Besides, the equation $(23)$ is used to find the equivalence of one model to the another.

\subsection{Modelling as a manipulator robot (relative coordinates)}

We report the procedure to obtain the dynamic model of the CBR as is usually done for a serial manipulator robot, i.e., $q_{1}$ is the position of the link attached at the base (in this case represented as the support leg) measured from the positive horizontal axis $(\mathrm{X}+)$ and $q_{2}$ is the angle measured from the extension line of the support leg to the second one (called the non-support, free or swing leg), as shown in Figure 3 . 


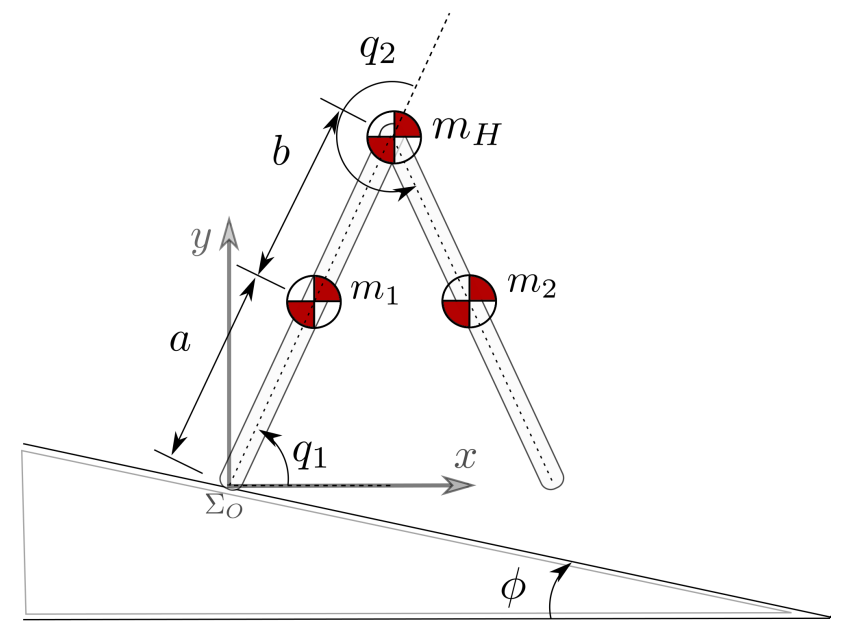

Figure 3: CBR with generalized coordinates as are normally taken for serial manipulator robots.

For the potential energy calculation, the vertical distances $h_{H}, h_{1}, h_{2}$, to the center of mass of the hip, leg 1 and leg $2\left(m_{H}, m_{1}, m_{2}\right.$ respectively) of the CBR measured from the support foot $\Sigma_{O}$ are

$$
h_{H}=l \sin \left(q_{1}\right), \quad h_{1}=a \sin \left(q_{1}\right), \quad h_{2}=l \sin \left(q_{1}\right)+b \sin \left(q_{1}+q_{2}\right),
$$

where $l=a+b$; and for the kinetic energy calculation, the position $\boldsymbol{x}_{H}, \boldsymbol{x}_{1}, \boldsymbol{x}_{2}$, of each center of mass with respect to the support foot $\Sigma_{O}$ are

$$
\begin{aligned}
\boldsymbol{x}_{H} & =\left[\begin{array}{l}
l \cos \left(q_{1}\right) \\
l \sin \left(q_{1}\right)
\end{array}\right], \quad \boldsymbol{x}_{1}=\left[\begin{array}{l}
a \cos \left(q_{1}\right) \\
a \sin \left(q_{1}\right)
\end{array}\right], \\
\boldsymbol{x}_{2}= & {\left[\begin{array}{l}
l \cos \left(q_{1}\right)+b \cos \left(q_{1}+q_{2}\right) \\
l \sin \left(q_{1}\right)+b \sin \left(q_{1}+q_{2}\right)
\end{array}\right] . }
\end{aligned}
$$

Now, by getting the time derivative from 29 we found the velocities of each center of mass given by

$$
\begin{gathered}
\boldsymbol{v}_{H}=\left[\begin{array}{c}
-l \sin \left(q_{1}\right) \dot{q}_{1} \\
l \cos \left(q_{1}\right) \dot{q}_{1}
\end{array}\right], \boldsymbol{v}_{1}=\left[\begin{array}{c}
-a \sin \left(q_{1}\right) \dot{q}_{1} \\
a \cos \left(q_{1}\right) \dot{q}_{1}
\end{array}\right], \\
\boldsymbol{v}_{2}=\left[\begin{array}{c}
-l \sin \left(q_{1}\right) \dot{q}_{1}-b \sin \left(q_{1}+q_{2}\right)\left(\dot{q}_{1}+\dot{q}_{2}\right) \\
l \cos \left(q_{1}\right) \dot{q}_{1}+b \cos \left(q_{1}+q_{2}\right)\left(\dot{q}_{1}+\dot{q}_{2}\right)
\end{array}\right] .
\end{gathered}
$$


Once we have obtained the velocities, it is possible to get the kinetic energy of the system given by

$$
\mathcal{K}=\frac{1}{2} m_{1}\left\|\boldsymbol{v}_{1}\right\|^{2}+\frac{1}{2} m_{2}\left\|\boldsymbol{v}_{2}\right\|^{2}+\frac{1}{2} m_{H}\left\|\boldsymbol{v}_{H}\right\|^{2},
$$

225 with $m=m_{1}=m_{2}$. In a similar way, to get the potential energy of the system, we substitute (28) into

$$
\mathcal{U}=m g h_{1}+m g h_{2}+m_{H} g h_{H} .
$$

Thus, the Lagrangian of the system is calculated as $L=\mathcal{K}-\mathcal{U}$. After applying the Euler-Lagrange equation of motion given by

$$
\frac{d}{d t}\left(\frac{\partial}{\partial \dot{\boldsymbol{q}}} L(\boldsymbol{q}, \dot{\boldsymbol{q}})\right)-\frac{\partial}{\partial \boldsymbol{q}} L(\boldsymbol{q}, \dot{\boldsymbol{q}})=\boldsymbol{\tau},
$$

the compact form

$$
M(\boldsymbol{q}) \ddot{\boldsymbol{q}}+C(\boldsymbol{q}, \dot{\boldsymbol{q}}) \dot{\boldsymbol{q}}+\boldsymbol{g}(\boldsymbol{q})=\boldsymbol{\tau}
$$

230 is obtained, where $\boldsymbol{\tau}$ is the vector of applied torques considering the whole dynamic of the robot, i.e., torques due to acceleration, gravity and reaction forces of subsequent links. Specifically, for the CBR model in 32 we have that

$$
\begin{gathered}
M(\boldsymbol{q})=\left[\begin{array}{cc}
m_{H} l^{2}+m\left(l^{2}+a^{2}+b^{2}\right)+2 m l b \cos \left(q_{2}\right) & m l b \cos \left(q_{2}\right)+m b^{2} \\
m l b \cos \left(q_{2}\right)+m b^{2} & m b^{2}
\end{array}\right], \\
C(\boldsymbol{q}, \dot{\boldsymbol{q}})=\left[\begin{array}{cc}
-m l b \sin \left(q_{2}\right) \dot{q}_{2} & -m l b \sin \left(q_{2}\right)\left(\dot{q}_{1}+\dot{q}_{2}\right) \\
m l b \sin \left(q_{2}\right) \dot{q}_{1} & 0
\end{array}\right],
\end{gathered}
$$

and

$$
\boldsymbol{g}(\boldsymbol{q})=\frac{\partial}{\partial \boldsymbol{q}} \mathcal{U}=\left[\begin{array}{l}
g_{1}(\boldsymbol{q}) \\
g_{2}(\boldsymbol{q})
\end{array}\right],
$$

235 with

$$
\begin{aligned}
& g_{1}(\boldsymbol{q})=m_{H} g l \cos \left(q_{1}\right)+m g a \cos \left(q_{1}\right)+m g\left(l \cos \left(q_{1}\right)+b \cos \left(q_{1}+q_{2}\right)\right), \\
& g_{2}(\boldsymbol{q})=m g b \cos \left(q_{1}+q_{2}\right) .
\end{aligned}
$$




\subsection{Modelling as in the biped robot literature (absolute coordinates)}

Now, we show the procedure for getting the dynamic model of the CBR system, but as is it usually done in the literature of biped robots (see [11] for further details), i.e., $\theta_{s}$ and $\theta_{n s}$ are the positions of the support and non-support $(\mathrm{Y}+)$, as shown in Figure 4

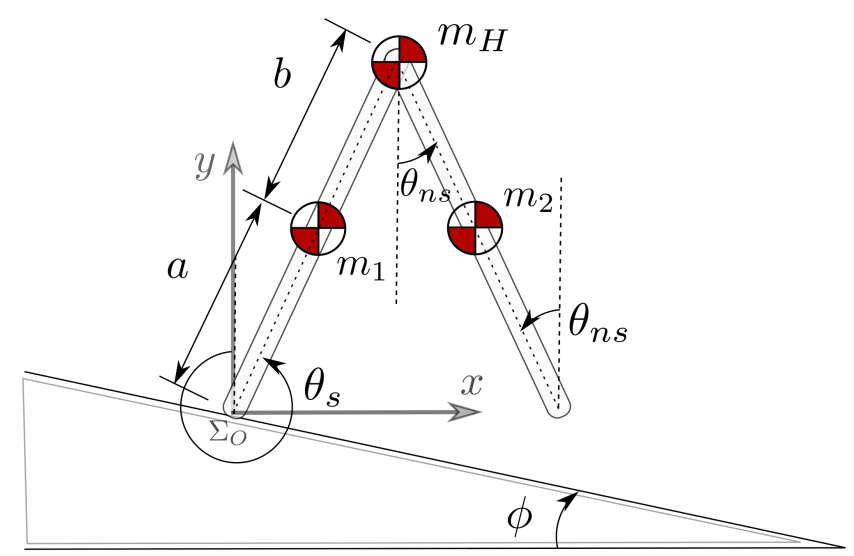

Figure 4: CBR with generalized coordinates as is usually done in the literature.

The vertical distances to each center of mass of the CBR for the potential energy calculation measured from the support foot $\Sigma_{O}$ are

$$
h_{H}=l \cos \left(\theta_{s}\right), \quad h_{1}=a \cos \left(\theta_{s}\right), \quad h_{2}=l \cos \left(\theta_{s}\right)-b \cos \left(\theta_{n s}\right) .
$$

The positions of each center of mass with respect to the support foot $\Sigma_{O}$ for the kinetic energy calculation are

$$
\begin{aligned}
& \boldsymbol{x}_{H}=\left[\begin{array}{c}
-l \sin \left(\theta_{s}\right) \\
l \cos \left(\theta_{s}\right)
\end{array}\right], \quad \boldsymbol{x}_{1}=\left[\begin{array}{c}
-a \sin \left(\theta_{s}\right) \\
a \cos \left(\theta_{s}\right)
\end{array}\right], \\
& \boldsymbol{x}_{2}=\left[\begin{array}{c}
-l \sin \left(\theta_{s}\right)+b \sin \left(\theta_{n s}\right) \\
l \cos \left(\theta_{s}\right)-b \cos \left(\theta_{n s}\right)
\end{array}\right] .
\end{aligned}
$$

Now, by getting the time derivative from (34) we find the velocities of each 
center of mass given by

$$
\begin{aligned}
& \boldsymbol{v}_{H}=\left[\begin{array}{c}
-l \cos \left(\theta_{s}\right) \dot{\theta}_{s} \\
-l \sin \left(\theta_{s}\right) \dot{\theta}_{s}
\end{array}\right], \quad \boldsymbol{v}_{1}=\left[\begin{array}{c}
-a \cos \left(\theta_{s}\right) \dot{\theta}_{s} \\
-a \sin \left(\theta_{s}\right) \dot{\theta}_{s}
\end{array}\right], \\
& \boldsymbol{v}_{2}=\left[\begin{array}{c}
-l \cos \left(\theta_{s}\right) \dot{\theta}_{s}+b \cos \left(\theta_{n s}\right) \dot{\theta}_{n s} \\
-l \sin \left(\theta_{s}\right) \dot{\theta}_{s}+b \sin \left(\theta_{n s}\right) \dot{\theta}_{n s}
\end{array}\right] .
\end{aligned}
$$

As done in the previous section, once the expression of the velocities are obtained, it is possible to get the kinetic energy of the system given by .

$$
\overline{\mathcal{K}}=\frac{1}{2} m_{1}\left\|\boldsymbol{v}_{1}\right\|^{2}+\frac{1}{2} m_{2}\left\|\boldsymbol{v}_{2}\right\|^{2}+\frac{1}{2} m_{H}\left\|\boldsymbol{v}_{H}\right\|^{2},
$$

250 with $m=m_{1}=m_{2}$. In a similar way, we use

$$
\overline{\mathcal{U}}=m g h_{1}+m g h_{2}+m_{H} g h_{H}
$$

to get the potential energy of the system. Now, the Lagrangian $\bar{L}=\overline{\mathcal{K}}-\overline{\mathcal{U}}$ of the system can be calculated. By using the Euler-Lagrange equation of motion given by

$$
\frac{d}{d t}\left(\frac{\partial}{\partial \dot{\boldsymbol{\theta}}} \bar{L}(\boldsymbol{\theta}, \dot{\boldsymbol{\theta}})\right)-\frac{\partial}{\partial \boldsymbol{\theta}} \bar{L}(\boldsymbol{\theta}, \dot{\boldsymbol{\theta}})=\overline{\boldsymbol{\tau}}
$$

it is possible to obtain a compact form equation given by

$$
\bar{M}(\boldsymbol{\theta}) \ddot{\boldsymbol{\theta}}+\bar{C}(\boldsymbol{\theta}, \dot{\boldsymbol{\theta}}) \dot{\boldsymbol{\theta}}+\overline{\boldsymbol{g}}(\boldsymbol{\theta})=\overline{\boldsymbol{\tau}},
$$

255 where

$$
\begin{gathered}
\bar{M}(\boldsymbol{\theta})=\left[\begin{array}{cc}
m_{H} l^{2}+m\left(l^{2}+a^{2}\right) & -m l b \cos \left(\theta_{s}-\theta_{n s}\right) \\
-m l b \cos \left(\theta_{s}-\theta_{n s}\right) & m b^{2}
\end{array}\right], \\
\bar{C}(\boldsymbol{\theta}, \dot{\boldsymbol{\theta}})=\left[\begin{array}{cc}
0 & -m l b \sin \left(\theta_{s}-\theta_{n s}\right) \dot{\theta}_{n s} \\
m l b \sin \left(\theta_{s}-\theta_{n s}\right) \dot{\theta_{s}} & 0
\end{array}\right],
\end{gathered}
$$

and

$$
\overline{\boldsymbol{g}}(\boldsymbol{\theta})=\frac{\partial}{\partial \boldsymbol{\theta}} \overline{\mathcal{U}}(\boldsymbol{\theta})=\left[\begin{array}{c}
-m_{H} g l \sin \left(\theta_{s}\right)-m g a \sin \left(\theta_{s}\right)-m g l \sin \left(\theta_{s}\right) \\
m g b \sin \left(\theta_{n s}\right)
\end{array}\right] .
$$


Notice that, in this case the torques $\bar{\tau}$ do not include the whole dynamic of the robot compared with the torques $\boldsymbol{\tau}$ obtained previously. Since $\theta_{s}$ and $\theta_{n s}$ are measured from fixed references, the links are considered decoupled, so that the reaction torque produced for link 2 (swing leg) into link 1 (stance leg) is not considered, as we will see in the next.

\subsection{Simulations}

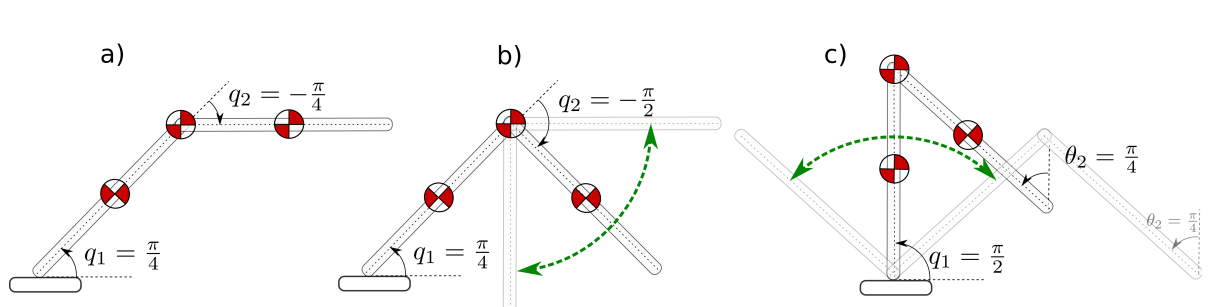

Figure 5: Different scenarios to evaluate the torques of dynamic models 32 and 37 .

The validation of previous dynamic models have already been considered in [9, 16] for serial robots, [6, 11, 13, 17] for planar biped robots and [18] for the 5 DOF planar biped robot of Section 5.

Now, according to theory explained in Section 3, here we perform some simulations for the motion of a CBR (which can be seen as a robot manipulator of 2 DOF) with the aim of comparing the torques in both models. We will show that, for the dynamic model of the CBR in equation (37), the torques needed to be applied to execute a motion are not complete for all links, except for the last one. The reason is that the torques $\overline{\boldsymbol{\tau}}$ do not take into account the reaction torque of subsequent links. The parameters of the model considered for simulation are: $m=5[\mathrm{~kg}], m_{H}=10[\mathrm{~kg}], a=b=0.5[\mathrm{~m}]$ and $g=9.81$ $275\left[\mathrm{~m} / \mathrm{s}^{2}\right]$.

There were considered three cases (shown in Figure 5): a) torques applied to keep the robot without moving, with the link 1 inclined 45 degrees $\left(q_{1}=\pi / 4\right.$ or $\left.\theta_{1}=-\pi / 4[\mathrm{rad}]\right)$ and the link 2 horizontal $\left(q_{2}=-\pi / 4\right.$ or $\left.\theta_{2}=\pi / 2[\mathrm{rad}]\right)$, which are shown in Figure 6. b) torques applied to keep the link 1 inclined 45 degrees 

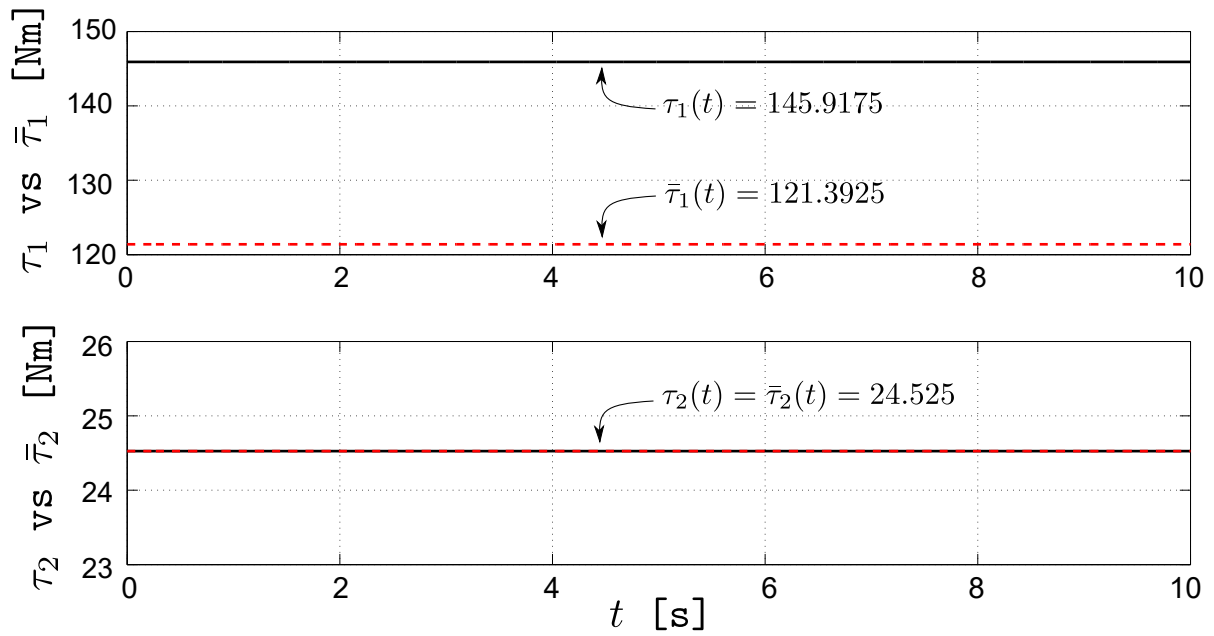

Figure 6: Torques applied to keep robot without moving. $\boldsymbol{\tau}$ in continuous line and $\overline{\boldsymbol{\tau}}$ in dashed line for dynamic models 32 and (37), respectively.

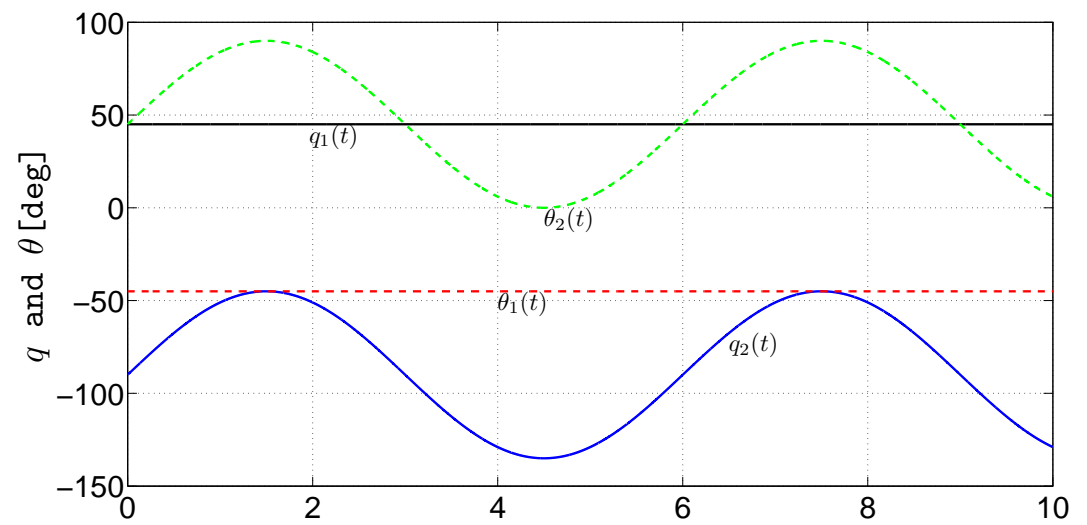

Figure 7: Time evolution of generalized coordinates $q$ and $\theta$ for case study (b).

$\left(q_{1}=\pi / 4[\mathrm{rad}]\right)$ without moving and moving the link 2 in a sinusoidal way with $\pi / 4[\mathrm{rad}]$ of amplitude, a period of $T=6[\mathrm{~s}]$ and starting from inclined position $\left(q_{2}=-\pi / 2[\mathrm{rad}]\right)$, whose result for the joint position and torques are shown in Figures 7 and 8 and c) torques applied to move the link 1 in a sinusoidal way with $\pi / 4[\mathrm{rad}]$ of amplitude, a period of $T=6[\mathrm{~s}]$, starting from vertical 

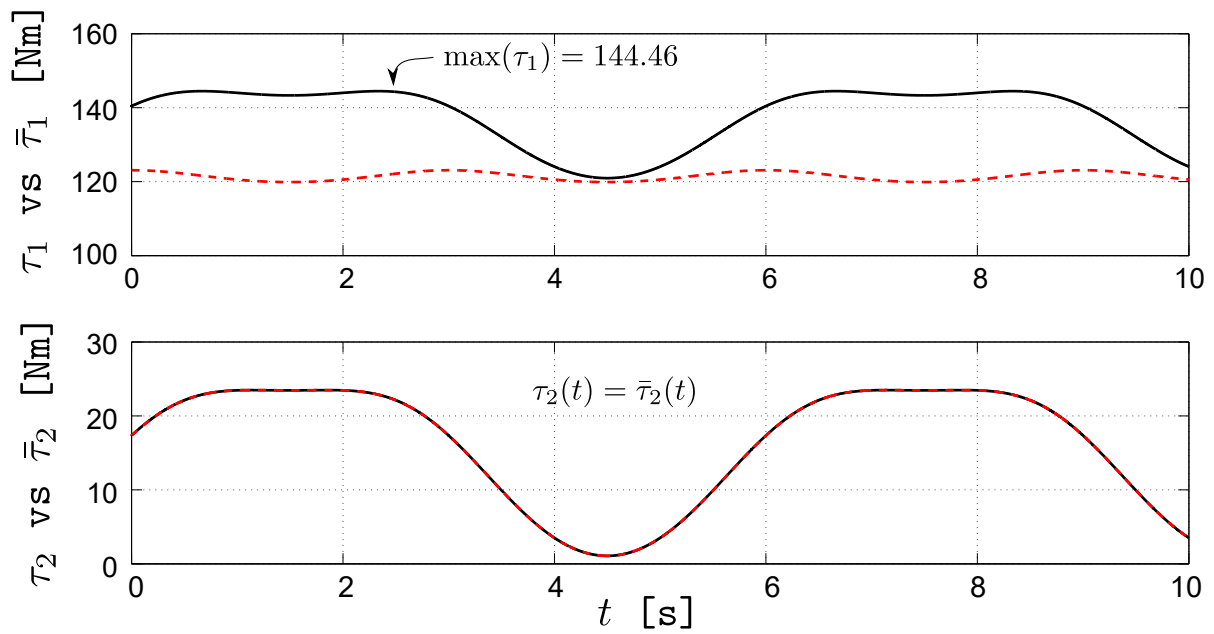

Figure 8: Torques applied to keep link 1 inclined 45 degrees without moving and link 2 continuously moving from downward vertical to horizontal position. $\boldsymbol{\tau}$ in continuous line and $\bar{\tau}$ in dashed line for dynamic models 32 and (37), respectively.

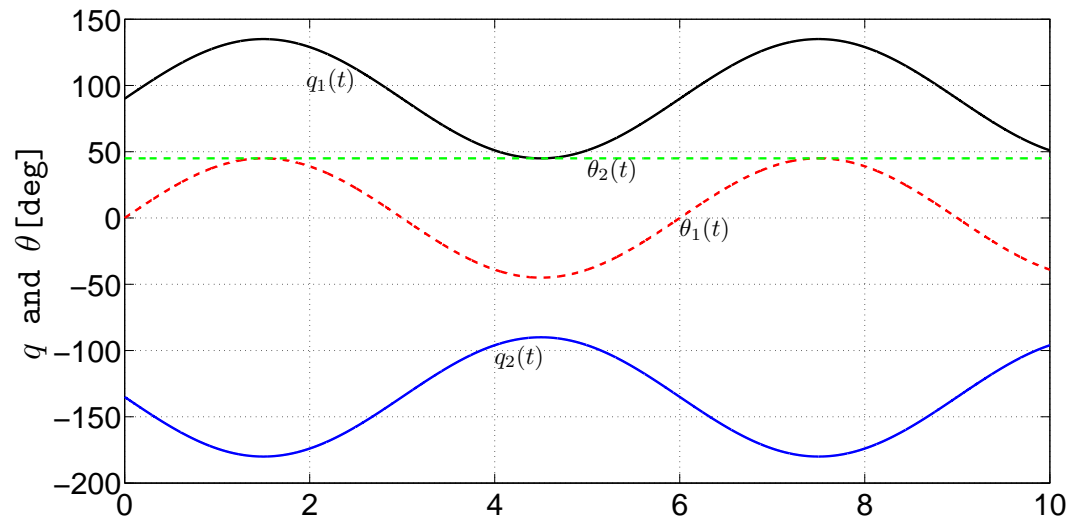

Figure 9: Time evolution of generalized coordinates $q$ and $\theta$ for case study (c).

position, and keeping the link 2 downward inclined without moving (inclined to $\theta_{2}=\pi / 4[\mathrm{rad}]$ for all $t$ ), which resulted in the joint positions and torques shown in Figures 9 and 10.

As observed in the case a), Figure 6 reveals that to keep the link 1 fixed, 

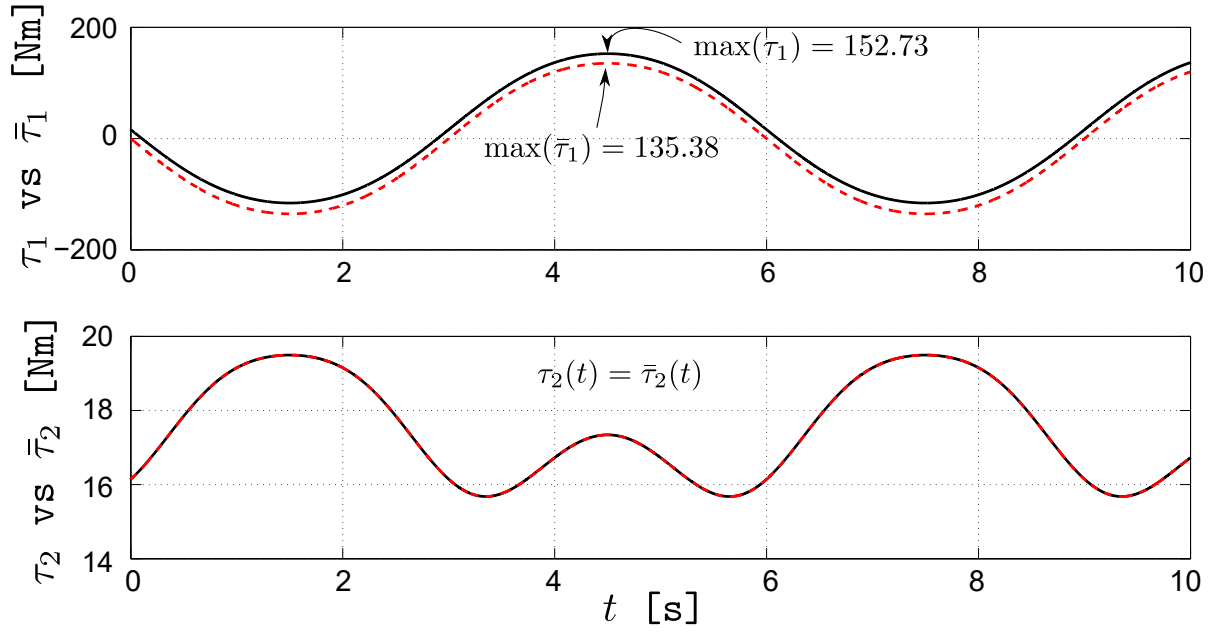

Figure 10: Torques applied to continuously move link 1 from -45 to 45 degrees from vertical position and keep link 2 fixed in an inclined position. $\boldsymbol{\tau}$ in continuous line and $\bar{\tau}$ in dashed line for dynamic models $(32)$ and $(37)$, respectively.

it is necessary to apply a torque $\tau_{1}$ of $145.9175[\mathrm{Nm}]$. However, we note that $\bar{\tau}_{1}$ for the link 1 is considerably smaller than $\tau_{1}\left(\bar{\tau}_{1}=121.3925[\mathrm{Nm}]\right)$, and if the torque needed to keep in position the link $2(24.525[\mathrm{Nm}])$ is added to $\bar{\tau}_{1}$, the result is the same as $\tau_{1}$, i.e. $\tau_{1}=\bar{\tau}_{1}+\bar{\tau}_{2}$. In fact, $\bar{\tau}_{1}$ does not consider the reaction torque applied from the link 2 to link 1 , it only considers gravity torques, and torques due to acceleration forces (since the robot is not moving the torques due to acceleration forces do not appear in this case).

For the case b), as seen in Figure 8 , the maximum torque $\tau_{1}$ applied for the link 1 is $144.46[\mathrm{Nm}$ ) (and not $145.9175[\mathrm{Nm}]$ ) this is because the forces and torques due to acceleration of the mass of link 2 (which do not appear in the first case) help to achieve the desired position. Moreover, gravity torque of link 2 and reaction torque from the link 2 are considered. The reaction torque into the link 1 due to link 2 is referred as the necessary torque applied by the actuator in the link 1 to compensate the torque applied by the actuator 2 at the link 2 to perform the desired motion of the second link. Nevertheless, $\bar{\tau}_{1}$ takes into consideration all torques except the one due to reaction torque from 
the link 2 .

Finally, for the case c) in Figure 10, we see how the shape of waves of the torques are similar. However, we can observe that the maximum torque $\tau_{1}$ needed to perform the motion of link 1 is bigger than $\bar{\tau}_{1}(152.73[\mathrm{Nm}]$ and 135.38 $[\mathrm{Nm}]$, respectively), this is because $\tau_{1}$ considers the reaction torque applied for the actuator of link 2 to keep it fixed into an inclined position. Note that in all figures, the torque applied to the last link (in this case the link 2) will be always the same (i.e., $\tau_{2}=\bar{\tau}_{2}$ ) for both models since there are not reaction torques at the end of link 2 from other links or external forces.

As a conclusion, we can say that, in order to get directly generalized forces $\tau$ that consider all forces and torques due to gravity, acceleration and reaction among links, we should use relative generalized coordinates $q$ in the Euler-Lagrange equation of motion. Equivalently, absolute generalized coordinates can be used but employing the equation (24).

\subsection{Relationship between the two CBR models}

Now, our purpose in this subsection is to show the equivalence between these two models, and also to explain how the input matrix, which is often used in the literature of biped robots, is obtained (see for example [19] or [17]). The dynamic model used in [19] and [17] is quite similar to the equation (37) but instead of using $\overline{\boldsymbol{\tau}}$ it is correctly used $B \boldsymbol{\tau}$, where $\boldsymbol{\tau}=\left[\begin{array}{ll}\tau_{1} & \tau_{2}\end{array}\right]^{T}$ are the torques at the support ankle and at the hip, that is,

$$
\bar{M}(\boldsymbol{\theta}) \ddot{\boldsymbol{\theta}}+\bar{C}(\boldsymbol{\theta}, \dot{\boldsymbol{\theta}}) \dot{\boldsymbol{\theta}}+\overline{\boldsymbol{g}}(\boldsymbol{\theta})=B \boldsymbol{\tau}
$$

We have already seen that it is not possible to get directly the applied torques $\boldsymbol{\tau}$ at the support ankle and at the hip, by taking as generalized coordinates $\theta_{s}$ and $\theta_{n s}$ to obtain the dynamic model of the CBR (we already know that the applied

330 torques $\overline{\boldsymbol{\tau}}$ do not take into account the whole dynamic of the robot and must not be considered to perform simulations or experimental tests). Nevertheless, 


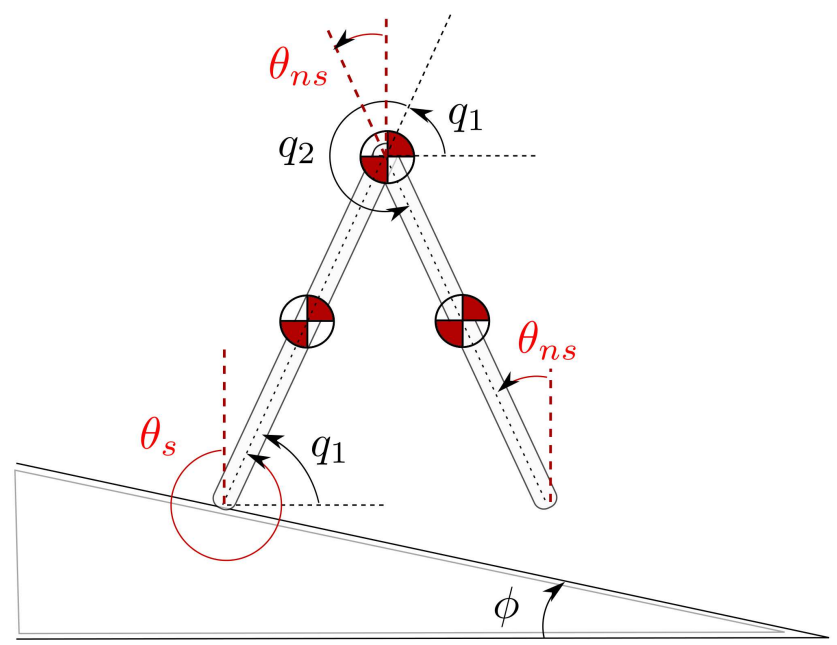

Figure 11: Comparison of generalized coordinates of both models.

when we use the generalized coordinates $q_{1}$ and $q_{2}$, the applied torques $\boldsymbol{\tau}$ at the support ankle and at the hip, are directly obtained.

So, it is clear that matrix $B$ in 41 is used for applying the torques $\boldsymbol{\tau}$ that consider the whole dynamic of the robot to the simpler dynamic model (37). To get the components of the matrix $B$ it is required to find the relation between the generalized coordinates used in both models, as was explained in Section 2. By doing a geometric analysis (see Figure 11), the relation between $\boldsymbol{\theta}$ and $\boldsymbol{q}$ is

$$
\begin{aligned}
& q_{1}=\theta_{s}-\frac{3}{2} \pi, \\
& q_{2}=\theta_{n s}-\theta_{s}+\pi,
\end{aligned}
$$

or

$$
\left[\begin{array}{l}
q_{1} \\
q_{2}
\end{array}\right]=\underbrace{\left[\begin{array}{cc}
1 & 0 \\
-1 & 1
\end{array}\right]}_{A}\left[\begin{array}{c}
\theta_{s} \\
\theta_{n s}
\end{array}\right]+\underbrace{\left[\begin{array}{c}
-\frac{3}{2} \pi \\
\pi
\end{array}\right]}_{\boldsymbol{b}} .
$$

where $A$ is the relation matrix between relative and absolute coordinates and $\boldsymbol{b}$ is a suitable constant vector. 
By getting the time derivative from 42 , the velocity relation is given by

$$
\dot{\boldsymbol{q}}=A \dot{\boldsymbol{\theta}}
$$

Then, by applying the time derivative to (43) we get the acceleration relation

$$
\ddot{\boldsymbol{q}}=A \ddot{\boldsymbol{\theta}}
$$

Once the relation between $\boldsymbol{\theta}$ and $\boldsymbol{q}$ has been obtained, we substitute 42,

$$
\begin{aligned}
M\left(\theta_{s}-\frac{3}{2} \pi, \theta_{n s}-\theta_{s}+\pi\right) A \ddot{\boldsymbol{\theta}}+C\left(\theta_{s}-\right. & \left.\frac{3}{2} \pi, \theta_{n s}-\theta_{s}+\pi, \dot{\theta}_{s}, \dot{\theta}_{n s}-\dot{\theta_{s}}\right) A \dot{\boldsymbol{\theta}} \\
& +\boldsymbol{g}\left(\theta_{s}-\frac{3}{2} \pi, \theta_{n s}-\theta_{s}+\pi,\right)=\boldsymbol{\tau} .
\end{aligned}
$$

As can be shown, the matrix

$$
M\left(\theta_{s}-\frac{3}{2} \pi, \theta_{n s}-\theta_{s}+\pi\right) A
$$

will not result necessarily into a symmetric matrix (which is a property of the matrix $M)$,

$$
\begin{aligned}
& M\left(\theta_{s}-\frac{3}{2} \pi, \theta_{n s}-\theta_{s}+\pi\right) A=\left[\begin{array}{ll}
\mu_{11} & \mu_{12} \\
\mu_{21} & \mu_{22}
\end{array}\right], \\
\mu_{11} & =m_{H} l^{2}+m\left(l^{2}+a^{2}+b^{2}\right)-m l b \cos \left(\theta_{n s}-\theta_{s}\right)-m b^{2}, \\
\mu_{12} & =-m l b \cos \left(\theta_{n s}-\theta_{s}\right)+m b^{2}, \\
\mu_{21} & =-m l b \cos \left(\theta_{n s}-\theta_{s}\right), \\
\mu_{22} & =m b^{2} .
\end{aligned}
$$

Therefore, it is necessary pre-multiply the matrix 46 by $A^{T}$ in order to recover the Lagrangian properties of the model. Thus, the whole equation 45 must be pre-multiplied by $A^{T}$ resulting

$$
\begin{array}{r}
A^{T} M\left(\theta_{s}-\frac{3}{2} \pi, \theta_{n s}-\theta_{s}+\pi\right) A \ddot{\boldsymbol{\theta}}+A^{T} C\left(\theta_{s}-\frac{3}{2} \pi, \theta_{n s}-\theta_{s}+\pi, \dot{\theta}_{s}, \dot{\theta}_{n s}-\dot{\theta_{s}}\right) A \dot{\boldsymbol{\theta}} \\
+A^{T} \boldsymbol{g}\left(\theta_{s}-\frac{3}{2} \pi, \theta_{n s}-\theta_{s}+\pi,\right)=A^{T} \boldsymbol{\tau},
\end{array}
$$


which is equivalent to the model in (41), and therefore equivalent to the model presented in the biped robot literature. So, we can identify

$$
\begin{aligned}
\bar{M}(\boldsymbol{\theta}) & =A^{T} M\left(\theta_{s}-\frac{3}{2} \pi, \theta_{n s}-\theta_{s}+\pi\right) A, \\
\bar{C}(\boldsymbol{\theta}, \dot{\boldsymbol{\theta}}) & =A^{T} C\left(\theta_{s}-\frac{3}{2} \pi, \theta_{n s}-\theta_{s}+\pi, \dot{\theta}_{s}, \dot{\theta}_{n s}-\dot{\theta_{s}}\right) A, \\
\overline{\boldsymbol{g}}(\boldsymbol{\theta}) & =A^{T} \boldsymbol{g}\left(\theta_{s}-\frac{3}{2} \pi, \theta_{n s}-\theta_{s}+\pi\right),
\end{aligned}
$$

355

and furthermore

$$
B=A^{T}
$$

where in this case $B$ is

$$
B=\left[\begin{array}{cc}
1 & -1 \\
0 & 1
\end{array}\right] \text {. }
$$

Thus, the input matrix $B$, the matrices $\bar{M}(\boldsymbol{\theta}), \bar{C}(\boldsymbol{\theta}, \dot{\theta})$ and vector $\bar{g}(\theta)$ with simplest elements defined by the left hand side of equation (37), i.e. equations $38-40$ and the torques $\tau$ from equation 32 (which considers the whole dynamic of the robot), can be related forming equation (41). Note that, it is not necessary to calculate both models. In practice, we just choose some generalized coordinates $\theta$ for obtaining the dynamic model of the planar biped (or serial) robot, then we get the relation of $\theta$ with the relative generalized coordinates $q$, to get the relation matrix $A$, and calculate the input matrix $B=A^{T}$. attached to the joints for obtaining the model equation (41).

Notice that this approach can also be used to calculate $M(q)$, $C(\boldsymbol{q}, \dot{\boldsymbol{q}})$ and $\boldsymbol{g}(\boldsymbol{q})$ directly from $\bar{M}(\boldsymbol{\theta}), \bar{C}(\boldsymbol{\theta}, \dot{\boldsymbol{\theta}})$ and $\overline{\boldsymbol{g}}(\boldsymbol{\theta})$ by means equations 25)-27, and vice versa by using equations $19-21$.

\section{Dynamic model of a 5 DOF biped robot}

Since planar biped robots are commonly composed by kinematic chains of high degrees of freedom and due to these kind of robots 
are not usually modelled by means of relative coordinates, the modelling of this class of robots is benefited by the equivalence procedure proposed in this work.

With the aim of showing the advantages of the proposed procedure, now, we show two different models for the SSP of the BipITLag biped robot built at the "Instituto Tecnológico de la Laguna" in México (further details of this biped robot are found in [18]).

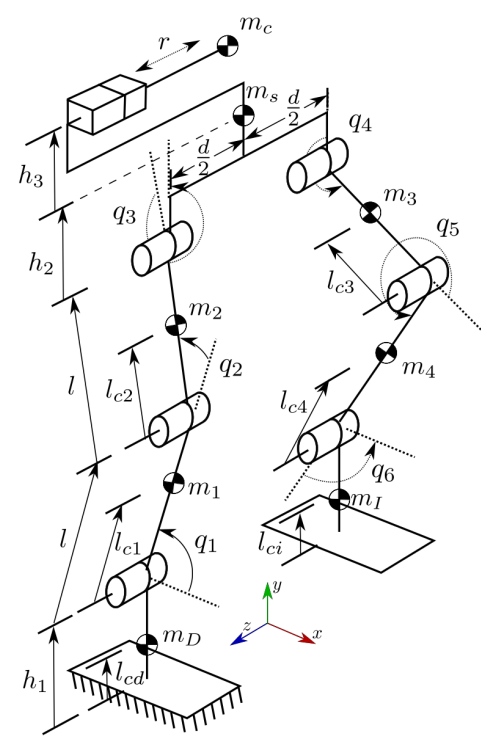

Figure 12: Kinematic model of the Bip-ITLag using relative coordinates.

By using the relative coordinates $\boldsymbol{q}=\left[q_{1}, q_{2}, q_{3}, q_{4}, q_{5}, q_{6}\right]^{T}$ shown in Figure 12, the position vectors of each center of mass (CoM) measured from the reference frame attached to the ankle are calculated 
and given by

$$
\begin{aligned}
\boldsymbol{x}_{m_{1}}= & {\left[\begin{array}{c}
l_{c 1} \cos \left(q_{1}\right) \\
l_{c 1} \sin \left(q_{1}\right) \\
0
\end{array}\right], \boldsymbol{x}_{m_{2}}=\left[\begin{array}{c}
l \cos \left(q_{1}\right)+l_{c 2} \cos \left(q_{1}+q_{2}\right) \\
l \sin \left(q_{1}\right)+l_{c 2} \sin \left(q_{1}+q_{2}\right) \\
0
\end{array}\right], } \\
\boldsymbol{x}_{m_{s}}= & {\left[\begin{array}{c}
l \cos \left(q_{1}\right)+l \cos \left(q_{1}+q_{2}\right) \\
l \sin \left(q_{1}\right)+l \sin \left(q_{1}+q_{2}\right)+h_{2} \\
-\frac{1}{2} d
\end{array}\right], \boldsymbol{x}_{m_{c}}=\left[\begin{array}{c}
l \cos \left(q_{1}\right)+l \cos \left(q_{1}+q_{2}\right) \\
l \sin \left(q_{1}\right)+l \sin \left(q_{1}+q_{2}\right)+h_{2}+h_{3} \\
-\frac{1}{2} d+r
\end{array}\right], }
\end{aligned}
$$

385

$$
\begin{gathered}
\boldsymbol{x}_{m_{3}}=\left[\begin{array}{c}
l \cos \left(q_{1}\right)+l \cos \left(q_{1}+q_{2}\right)+\left(l-l_{c 3}\right) \cos \left(q_{4}+\frac{\pi}{2}\right) \\
l \sin \left(q_{1}\right)+l \sin \left(q_{1}+q_{2}\right)+\left(l-l_{c 3}\right) \sin \left(q_{4}+\frac{\pi}{2}\right) \\
-d
\end{array}\right], \\
\boldsymbol{x}_{m_{4}}=\left[\begin{array}{c}
l \cos \left(q_{1}\right)+l \cos \left(q_{1}+q_{2}\right)+l \cos \left(q_{4}+\frac{\pi}{2}\right)+\left(l-l_{c 4}\right) \cos \left(q_{4}+q_{5}+\frac{\pi}{2}\right) \\
l \sin \left(q_{1}\right)+l \sin \left(q_{1}+q_{2}\right)+l \sin \left(q_{4}+\frac{\pi}{2}\right)+\left(l-l_{c 4}\right) \sin \left(q_{4}+q_{5}+\frac{\pi}{2}\right) \\
-d
\end{array}\right], \\
\boldsymbol{x}_{m_{I}}=\left[\begin{array}{c} 
\\
l \cos \left(q_{1}\right)+l \cos \left(q_{1}+q_{2}\right)+l \cos \left(q_{4}+\frac{\pi}{2}\right)+l \cos \left(q_{4}+q_{5}+\frac{\pi}{2}\right) \\
l \sin \left(q_{1}\right)+l \sin \left(q_{1}+q_{2}\right)+l \sin \left(q_{4}+\frac{\pi}{2}\right)+l \sin \left(q_{4}+q_{5}+\frac{\pi}{2}\right)-h_{1}+l_{c i} \\
-d
\end{array}\right] .
\end{gathered}
$$

In a similar way as for the CBR, by using the Euler-Lagrange equation of motion, a compact form of the Bip-ITLag robot dynamic model is obtained. i.e.,

$$
M(\boldsymbol{q}) \ddot{\boldsymbol{q}}+C(\boldsymbol{q}, \dot{\boldsymbol{q}}) \dot{\boldsymbol{q}}+\boldsymbol{g}(\boldsymbol{q})=\boldsymbol{\tau}
$$

where the respective matrices are defined by

$$
M(\boldsymbol{q})=\left[\begin{array}{ccccc}
m_{11} & m_{12} & m_{13} & m_{14} & 0 \\
m_{12} & m_{22} & m_{23} & m_{24} & 0 \\
m_{13} & m_{23} & m_{33} & m_{34} & 0 \\
m_{14} & m_{24} & m_{34} & m_{44} & 0 \\
0 & 0 & 0 & 0 & m_{55}
\end{array}\right],
$$


with

$$
\begin{aligned}
m_{11}= & K_{A}+K_{B}+2 K_{F} \cos \left(q_{2}\right) \\
m_{12}= & K_{B}+K_{F} \cos \left(q_{2}\right) \\
m_{13}= & -K_{G} \sin \left(q_{1}-q_{4}\right)-K_{G} \sin \left(q_{1}+q_{2}-q_{4}\right)-K_{H} \sin \left(q_{1}-q_{4}-q_{5}\right) \\
& -K_{H} \sin \left(q_{1}+q_{2}-q_{4}-q_{5}\right), \\
m_{14}= & -K_{H}\left(\sin \left(q_{1}-q_{4}-q_{5}\right)+\sin \left(q_{1}+q_{2}-q_{4}-q_{5}\right)\right), \\
m_{22}= & K_{B}, \\
m_{23}= & -K_{G} \sin \left(q_{1}+q_{2}-q_{4}\right)-K_{H} \sin \left(q_{1}+q_{2}-q_{4}-q_{5}\right), \\
m_{24}= & -K_{H} \sin \left(q_{1}+q_{2}-q_{4}-q_{5}\right), \\
m_{33}= & K_{C}+K_{D}-2 K_{H} \cos \left(q_{5}\right), \\
m_{34}= & K_{D}-K_{H} \cos \left(q_{5}\right), \\
m_{44}= & K_{D}, \\
m_{55}= & m_{c},
\end{aligned}
$$

$$
C(\boldsymbol{q}, \dot{\boldsymbol{q}})=\left[\begin{array}{ccccc}
c_{11} & c_{12} & c_{13} & c_{14} & 0 \\
c_{21} & 0 & c_{23} & c_{24} & 0 \\
c_{31} & c_{32} & c_{33} & c_{34} & 0 \\
c_{41} & c_{42} & c_{43} & 0 & 0 \\
0 & 0 & 0 & 0 & 0
\end{array}\right]
$$

\section{whose elements are given by}

$$
\begin{aligned}
c_{11}= & -K_{F} \sin \left(q_{2}\right) \dot{q}_{2}, \\
c_{12}= & -K_{F} \sin \left(q_{2}\right)\left(\dot{q}_{1}+\dot{q}_{2}\right), \\
c_{13}= & K_{G} \cos \left(q_{1}-q_{4}\right) \dot{q}_{4}+K_{G} \cos \left(q_{1}+q_{2}-q_{4}\right) \dot{q}_{4}+K_{H} \cos \left(q_{1}-q_{4}-q_{5}\right)\left(\dot{q}_{4}+\dot{q}_{5}\right) \\
& +K_{H} \cos \left(q_{1}+q_{2}-q_{4}-q_{5}\right)\left(\dot{q}_{4}+\dot{q}_{5}\right), \\
c_{14}= & K_{H}\left(\cos \left(q_{1}-q_{4}-q_{5}\right)+\cos \left(q_{1}+q_{2}-q_{4}-q_{5}\right)\right)\left(\dot{q}_{4}+\dot{q}_{5}\right), \\
c_{21}= & K_{F} \sin \left(q_{2}\right) \dot{q}_{1}, \\
c_{23}= & K_{G} \cos \left(q_{1}+q_{2}-q_{4}\right) \dot{q}_{4}+K_{H} \cos \left(q_{1}+q_{2}-q_{4}-q_{5}\right)\left(\dot{q}_{4}+\dot{q}_{5}\right),
\end{aligned}
$$




$$
\begin{aligned}
c_{24}= & K_{H} \cos \left(q_{1}+q_{2}-q_{4}-q_{5}\right)\left(\dot{q}_{4}+\dot{q}_{5}\right), \\
c_{31}= & -K_{G} \cos \left(q_{1}-q_{4}\right) \dot{q}_{1}-K_{H} \cos \left(q_{1}-q_{4}-q_{5}\right) \dot{q}_{1}-K_{G} \cos \left(q_{1}+q_{2}-q_{4}\right)\left(\dot{q}_{1}+\dot{q}_{2}\right) \\
& -K_{H} \cos \left(q_{1}+q_{2}-q_{4}-q_{5}\right)\left(\dot{q}_{1}+\dot{q}_{2}\right), \\
c_{32}= & -\left(K_{G} \cos \left(q_{1}+q_{2}-q_{4}\right)+K_{H} \cos \left(q_{1}+q_{2}-q_{4}-q_{5}\right)\right)\left(\dot{q}_{1}+\dot{q}_{2}\right), \\
c_{33}= & K_{H} \sin \left(q_{5}\right) \dot{q}_{5}, \\
c_{34}= & K_{H} \sin \left(q_{5}\right)\left(\dot{q}_{4}+\dot{q}_{5}\right), \\
c_{41}= & -K_{H}\left(\cos \left(q_{1}-q_{4}-q_{5}\right) \dot{q}_{1}+\cos \left(q_{1}+q_{2}-q_{4}-q_{5}\right)\left(\dot{q}_{1}+\dot{q}_{2}\right)\right), \\
c_{42}= & -K_{H} \cos \left(q_{1}+q_{2}-q_{4}-q_{5}\right)\left(\dot{q}_{1}+\dot{q}_{2}\right), \\
c_{43}= & -K_{H} \sin \left(q_{5}\right) \dot{q}_{4}, \\
\text { and } &
\end{aligned}
$$

$$
\boldsymbol{g}(\boldsymbol{q})=\left[\begin{array}{c}
g\left(K_{L} \cos \left(q_{1}\right)+K_{M} \cos \left(q_{1}+q_{2}\right)\right) \\
K_{M} g \cos \left(q_{1}+q_{2}\right) \\
g\left(K_{N} \sin \left(q_{4}\right)+K_{O} \sin \left(q_{4}+q_{5}\right)\right) \\
K_{O} g \sin \left(q_{4}+q_{5}\right) \\
0
\end{array}\right]
$$

In order to reduce the dimension of the expressions some constants were defined as follows:

$$
\begin{aligned}
& K_{A}=l_{c 1}^{2} m_{1}+l^{2}\left(m_{2}+m_{c}+m_{s}+m_{3}+m_{4}+m_{I}\right)+I_{1}, \\
& K_{B}=l_{c 2}^{2} m_{2}+l^{2}\left(m_{s}+m_{c}+m_{3}+m_{4}+m_{I}\right)+I_{2}, \\
& K_{C}=\left(l-l_{c 3}\right)^{2} m_{3}+l^{2}\left(m_{4}+m_{I}\right)+I_{3}, \\
& K_{D}=\left(l-l_{c 4}\right)^{2} m_{4}+l^{2} m_{I}+I_{4} \\
& K_{F}=l l_{c 2} m_{2}+l^{2}\left(m_{s}+m_{c}+m_{3}+m_{4}+m_{I}\right) \\
& K_{G}=-l\left(l-l_{c 3}\right) m_{3}-l^{2}\left(m_{4}+m_{I}\right) \\
& K_{H}=-l\left(l-l_{c 4}\right) m_{4}-l^{2} m_{I}, \\
& K_{L}=m_{1} l_{c 1}+l\left(m_{2}+m_{c}+m_{s}+m_{3}+m_{4}+m_{I}\right), \\
& K_{M}=m_{2} l_{c 2}+l\left(m_{s}+m_{c}+m_{3}+m_{4}+m_{I}\right)
\end{aligned}
$$




$$
\begin{aligned}
& K_{N}=-m_{3}\left(l-l_{c 3}\right)-l\left(m_{4}+m_{I}\right), \\
& K_{O}=-m_{4}\left(l-l_{c 4}\right)-l m_{I},
\end{aligned}
$$

where $m_{I}, m_{s}$ and $m_{c}$ are the masses of the free foot, support foot, and hip, respectively. $m_{1}, m_{2}, m_{3}$ and $m_{4}$ are the masses of the tibias and femurs of the support and free legs. $l_{c 1}, l_{c 2}, l_{c 3}$ and $l_{c 4}$ are the distances to the CoM from each joint, as shown in Figure 12 and 13.

\subsection{Modelling using absolute coordinates}

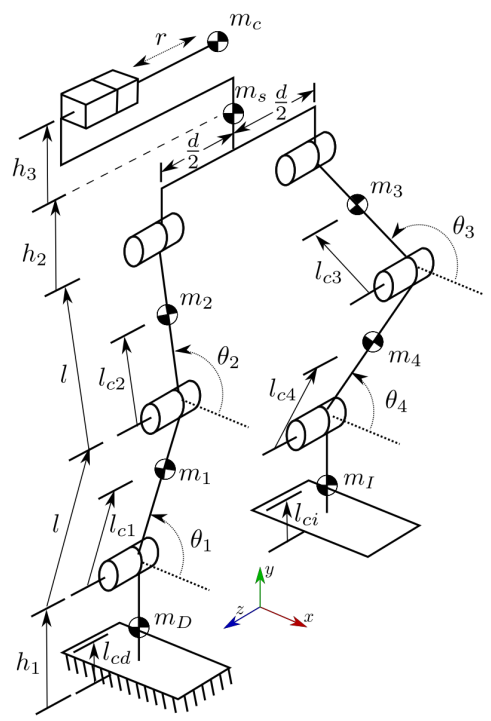

Figure 13: Kinematic model of the Bip-ITLag using absolute coordinates. $\theta_{1}, \theta_{2}, \theta_{3}$ and $\theta_{4}$ shown in Figure 13 , the positions of CoM of each link 
measured from support ankle are given by

$$
\begin{gathered}
\boldsymbol{x}_{m_{1}}=\left[\begin{array}{c}
l_{c 1} \cos \left(\theta_{1}\right) \\
l_{c 1} \sin \left(\theta_{1}\right) \\
0
\end{array}\right], \boldsymbol{x}_{m_{2}}=\left[\begin{array}{c}
l \cos \left(\theta_{1}\right)+l_{c 2} \cos \left(\theta_{2}\right) \\
l \sin \left(\theta_{1}\right)+l_{c 2} \sin \left(\theta_{2}\right) \\
0
\end{array}\right], \\
\boldsymbol{x}_{m_{s}}=\left[\begin{array}{c}
l \cos \left(\theta_{1}\right)+l \cos \left(\theta_{2}\right) \\
l \sin \left(\theta_{1}\right)+l \sin \left(\theta_{2}\right)+h_{2} \\
-\frac{1}{2} d
\end{array}\right], \boldsymbol{x}_{m_{c}}=\left[\begin{array}{c}
l \cos \left(\theta_{1}\right)+l \cos \left(\theta_{2}\right) \\
l \sin \left(\theta_{1}\right)+l \sin \left(\theta_{2}\right)+h_{2}+h_{3} \\
-\frac{1}{2} d+r
\end{array}\right], \\
\boldsymbol{x}_{m_{3}}=\left[\begin{array}{c}
l \cos \left(\theta_{1}\right)+l \cos \left(\theta_{2}\right)-\left(l-l_{c 3}\right) \cos \left(\theta_{3}\right) \\
l \sin \left(\theta_{1}\right)+l \sin \left(\theta_{2}\right)-\left(l-l_{c 3}\right) \sin \left(\theta_{3}\right) \\
-d
\end{array}\right], \\
\boldsymbol{x}_{m_{4}}=\left[\begin{array}{c}
l \cos \left(\theta_{1}\right)+l \cos \left(\theta_{2}\right)-l \cos \left(\theta_{3}\right)-\left(l-l_{c 4}\right) \cos \left(\theta_{4}\right) \\
l \sin \left(\theta_{1}\right)+l \sin \left(\theta_{2}\right)-l \sin \left(\theta_{3}\right)-\left(l-l_{c 4}\right) \sin \left(\theta_{4}\right) \\
-d
\end{array}\right],
\end{gathered}
$$

410

$$
\boldsymbol{x}_{m_{I}}=\left[\begin{array}{c}
l \cos \left(\theta_{1}\right)+l \cos \left(\theta_{2}\right)-l \cos \left(\theta_{3}\right)-l \cos \left(\theta_{4}\right) \\
l \sin \left(\theta_{1}\right)+l \sin \left(\theta_{2}\right)-l \sin \left(\theta_{3}\right)-l \sin \left(\theta_{4}\right)-h_{1}+l_{c i} \\
-d
\end{array}\right]
$$

and after applying the Euler-Lagrange equation of motion, the dynamic model in a compact form is defined as

$$
\bar{M}(\boldsymbol{\theta}) \ddot{\boldsymbol{\theta}}+\bar{C}(\boldsymbol{\theta}, \dot{\boldsymbol{\theta}}) \dot{\boldsymbol{\theta}}+\overline{\boldsymbol{g}}(\boldsymbol{\theta})=\overline{\boldsymbol{\tau}},
$$

with elements given by

$$
\bar{M}(\boldsymbol{\theta})=\left[\begin{array}{ccccc}
K_{A} & K_{F} \cos \left(\theta_{12}\right) & K_{G} \cos \left(\theta_{13}\right) & K_{H} \cos \left(\theta_{14}\right) & 0 \\
K_{F} \cos \left(\theta_{12}\right) & K_{B} & K_{G} \cos \left(\theta_{23}\right) & K_{H} \cos \left(\theta_{24}\right) & 0 \\
K_{G} \cos \left(\theta_{13}\right) & K_{G} \cos \left(\theta_{23}\right) & K_{C} & -K_{H} \cos \left(\theta_{34}\right) & 0 \\
K_{H} \cos \left(\theta_{14}\right) & K_{H} \cos \left(\theta_{24}\right) & -K_{H} \cos \left(\theta_{34}\right) & K_{D} & 0 \\
0 & 0 & 0 & 0 & m_{c}
\end{array}\right],
$$




$$
\bar{C}(\boldsymbol{\theta}, \dot{\boldsymbol{\theta}})=\left[\begin{array}{ccccc}
0 & K_{F} \sin \left(\theta_{12}\right) \dot{\theta_{2}} & K_{G} \sin \left(\theta_{13}\right) \dot{\theta_{3}} & K_{H} \sin \left(\theta_{14}\right) \dot{\theta_{4}} & 0 \\
-K_{F} \sin \left(\theta_{12}\right) \dot{\theta_{1}} & 0 & K_{G} \sin \left(\theta_{23}\right) \dot{\theta_{3}} & K_{H} \sin \left(\theta_{24}\right) \dot{\theta_{4}} & 0 \\
-K_{G} \sin \left(\theta_{13}\right) \dot{\theta_{1}} & -K_{G} \sin \left(\theta_{23}\right) \dot{\theta_{2}} & 0 & -K_{H} \sin \left(\theta_{34}\right) \dot{\theta_{4}} & 0 \\
-K_{H} \sin \left(\theta_{14}\right) \dot{\theta_{1}} & -K_{H} \sin \left(\theta_{24}\right) \dot{\theta_{2}} & K_{H} \sin \left(\theta_{34}\right) \dot{\theta_{3}} & 0 & 0 \\
0 & 0 & 0 & 0 & 0
\end{array}\right],(50
$$

and

$$
\overline{\boldsymbol{g}}(\boldsymbol{\theta})=\left[\begin{array}{c}
K_{L} g \cos \left(\theta_{1}\right) \\
K_{M} g \cos \left(\theta_{2}\right) \\
K_{N} g \cos \left(\theta_{3}\right) \\
K_{O} g \cos \left(\theta_{4}\right) \\
0
\end{array}\right] .
$$

where $\theta_{i j}=\theta_{i}-\theta_{j}$. The constants $K_{A}, \ldots, K_{O}$ were defined previously.

It is clear that $\bar{M}(\boldsymbol{\theta}), \bar{C}(\boldsymbol{\theta}, \dot{\boldsymbol{\theta}})$ and $\overline{\boldsymbol{g}}(\boldsymbol{\theta})$ have simpler elements than $M(\boldsymbol{q}), C(\boldsymbol{q}, \dot{\boldsymbol{q}})$ and $\boldsymbol{g}(\boldsymbol{q})$, and the longer the kinematic chain, the longer the complexity of elements of the model where relative coordinates are used. Thus, in order to implement the model in microprocessorbased systems, such as embedded systems, or where online optimization algorithms that use the dynamic model are used, it is a reasonable good idea to use absolute coordinates to get the dynamic model. However, as we have seen before the torque $\bar{\tau}$ does not take into account the reaction forces between links. So, it is necessary to use the equivalence with $\tau$.

\subsection{Relation between the two Bip-ITLag robot models}

It is convenient to use the matrices with the simplest elements of the dynamic model (48), but using the torques of equation (47). For doing this, it is enough to know the relation between the generalized coordinates used for getting the models (47) and (48). This relation 
is given by

$$
\left[\begin{array}{c}
q_{1} \\
q_{2} \\
q_{4} \\
q_{5} \\
r
\end{array}\right]=\left[\begin{array}{l}
\theta_{1} \\
\theta_{2}-\theta_{1} \\
\theta_{3}+\frac{\pi}{2} \\
\theta_{4}-\theta_{3} \\
r
\end{array}\right]=\underbrace{\left[\begin{array}{ccccc}
1 & 0 & 0 & 0 & 0 \\
-1 & 1 & 0 & 0 & 0 \\
0 & 0 & 1 & 0 & 0 \\
0 & 0 & -1 & 1 & 0 \\
0 & 0 & 0 & 0 & 1
\end{array}\right]}_{A}\left[\begin{array}{c}
\theta_{1} \\
\theta_{2} \\
\theta_{3} \\
\theta_{4} \\
r
\end{array}\right]+\underbrace{\left[\begin{array}{c}
0 \\
0 \\
\frac{\pi}{2} \\
0 \\
0
\end{array}\right]}_{b} \cdot(52)
$$

Therefore, the velocity and acceleration relations are given by

$$
\begin{aligned}
\dot{\boldsymbol{q}} & =A \dot{\boldsymbol{\theta}}, \\
\ddot{\boldsymbol{q}} & =A \ddot{\boldsymbol{\theta}},
\end{aligned}
$$

respectively. By using the relation matrix $A$ given in $(52)$ and equations (49)-(51), the relation (24) given between the model obtained in absolute coordinates and the model expressed in relative coordinates is found, and vice versa. It is worthwhile to notice that equation (23) is only required to compute the input matrix $B$, so that we can have

$$
\bar{M}(\boldsymbol{\theta}) \ddot{\boldsymbol{\theta}}+\bar{C}(\boldsymbol{\theta}, \dot{\boldsymbol{\theta}}) \dot{\boldsymbol{\theta}}+\overline{\boldsymbol{g}}(\boldsymbol{\theta})=B \boldsymbol{\tau}
$$

where

$$
B=A^{T}=\left[\begin{array}{ccccc}
1 & -1 & 0 & 0 & 0 \\
0 & 1 & 0 & 0 & 0 \\
0 & 0 & 1 & -1 & 0 \\
0 & 0 & 0 & 1 & 0 \\
0 & 0 & 0 & 0 & 1
\end{array}\right]
$$

which is invertible.

\section{Conclusions}

In this paper, a procedure to find the equivalence among different dynamic model representations of planar biped (or serial) robots was shown. These 
model representations come from using any particular generalized coordinates. Without loss of generality, the absolute coordinate-based model and the relative coordinate-based model were studied. We have introduced a novel procedure to find the relation between a dynamic model and another one (see equations $(19,, 20,, 21$ and $(23)$ ). This procedure is useful for the development of control laws and implementation of the dynamic model in embedded platforms by reducing the number of calculations, and by reducing the computing time when the dynamic model is used in optimization problems, the design of control laws, and so on.

The CBR dynamic model was used as example to explain such a procedure, where we clearly showed how to obtain the input matrix that appears in the models reported in the biped robot literature. Simulations showed that for getting directly torques vector $\tau$ that considers all forces and torques due to gravity, acceleration and reaction among links, relative generalized coordinates in the Euler-Lagrange equation of motion should be used. Absolute coordinates were helpful to obtain a dynamic model with matrices with simpler elements compared with the ones derived from relatives coordinates. Furthermore, we showed that no matter which generalized coordinates are used to obtain the dynamic models, there is always an equivalence among them by using a particular input matrix. In addition, the comparison between two dynamic models of the SSP of a 5 DOF biped robot was performed in order to show the advantages of our approach, where biped robots with high DOF are modelled.

\section{Acknowledgements}

This work is partially supported by CONACyT under Grant no. 134534 and by TecNM. 
[1] S. Collins, M. Wisse, A. Ruina, A three-dimensional passive-dynamic walking robot with two legs and knees, Int. J. of Robotics Research 20 (7) (2001) $607-6015$.

[2] M. Gomes, A. Ruina, Walking model with no energy cost, Phys. Rev. E 83, 03290183 (3) (2011) 35-39.

[3] S. Collins, A. Ruina, R. Tedrake, M. Wisse, Efficient bipedal robots based on passive dynamic walkers, Science 307 (5712) (2005) 1082-1085.

[4] J. Rouhollah, L. L. Flynn, A. Hellum, R. Mukherjee, Energy-conserving gaits for point-foot planar bipeds: A five-dof case study, in: ASME 2013 Dynamic Systems and Control Conference, San Diego, California, USA, 2013.

[5] F. Plestan, J. W. Grizzle, E. R. Westervelt, G. Abba, Stable walking of a 7-dof biped robot, IEEE Transactions on Robotics and Automation 19 (4) (2003) 653-668.

[6] C. Chevallereau, G. Abba, Y. Aoustin, E. R. W. F. Plestan, C. Canudas, J. Grizzle, Rabbit: a testbed for advanced control theory, IEEE Control Systems Magazine 23(5) (2003) 57-79.

[7] R. W. Sinnet, A. D. Ames, 2d bipedal walking with knees and feet: A hybrid control approach, in: Decision and Control, 2009 held jointly with the 2009 28th Chinese Control Conference. CDC/CCC 2009. Proceedings of the 48th IEEE Conference on, 2009, pp. 3200-3207.

[8] K. Sreenath, H.-W. Park, J. Grizzle, Design and experimental implementation of a compliant hybrid zero dynamics controller with active force control for running on mabel, in: Robotics and Automation (ICRA), 2012 IEEE International Conference on, Saint Paul, MN, USA, 2012, pp. 51-56. 
[9] B. Siciliano, L. Sciavicco, L. Villani, G. Oriolo, Robotics Modelling, Planning and Control, Springer-Verlag, 2009.

[10] J. A. Acosta, R. Ortega, A. Astolfi, A. Mahindrakar, Interconnection and damping assignment passivity-based control of mechanical systems with underactuation degree one, IEEE Transactions on Automatic Control 50 (12) (2005) 1936-1955.

[11] A. Goswami, B. Thuilot, B. Espiau, A study of the passive gait of a compass-like biped robot: Symmetry and chaos, Int. J. of Robotics Research 17 (12) (1998) 1282-1301.

[12] M. Spong, Passivity based control of the compass gait biped, in: IFAC World Congress, Beijing, China, 1999, pp. 12-24.

[13] M. Spong, F. Bullo, Controlled symmetries and passive walking, in: IFAC World Congress, Barcelona, Spain, 2002, pp. 557-562.

[14] A. Olenšek, Z. Matjačić, Two-level control strategy of an eight link biped walking model, Simulation Modelling Practice and Theory 19 (1) (2011) $133-147$.

[15] S. Yahya, M. Moghavvemi, H. A. Mohamed, Geometrical approach of planar hyper-redundant manipulators: Inverse kinematics, path planning and workspace, Simulation Modelling Practice and Theory 19 (1) (2011) 406422.

[16] R. Kelly, V. Santibanez, A. Loría, Control of Robot Manipulators in Joint Space, Springer London, 2005.

[17] J. Holm, M. W. Spong, Kinetic energy shaping for gait regulation of underactuated bipeds, in: IEEE International Conference on Control Applications, 2008, pp. 1232-1238.

[18] V. De-León-Gómez, J. A. Pámanes, V. Santibanez, Experimental evaluation of the real-time walking of a 5 dof biped robot with a gait based on 
cycloidal motions, Revista Iberoamericana de Automática e Informática Industrial RIAI 12 (04) (2015) 408-418.

${ }_{525}$ [19] M. Spong, F. Bullo, Controlled symmetries and passive walking, IEEE Trans. Automatic Control 50 (7) (2005) 1025-1031.

Appendix. Proof of power equivalence between model (9) and (18).

The dynamic model of a mechanical system can be represented in a compact form as

$$
M(\boldsymbol{q}) \ddot{\boldsymbol{q}}+C(\boldsymbol{q}, \dot{\boldsymbol{q}}) \dot{\boldsymbol{q}}+\boldsymbol{g}(\boldsymbol{q})=\boldsymbol{\tau},
$$

where $\boldsymbol{q}, \dot{\boldsymbol{q}}, \ddot{\boldsymbol{q}} \in \mathbb{R}^{n}$, are the joint position, velocity and acceleration vectors, respectively, $M(\boldsymbol{q})=M(\boldsymbol{q})^{T}>0$ is the inertia matrix, $C(\boldsymbol{q}, \dot{\boldsymbol{q}})$ is the matrix of Coriolis and centrifugal forces, $\boldsymbol{g}(\boldsymbol{q})=\frac{\partial \mathcal{U}(\boldsymbol{q})}{\partial \boldsymbol{q}}$ is the vector of gravitational torques and $\tau \in \mathbb{R}^{n}$ is the vector of applied torques. Furthermore, the energy of the mechanical system A.1 can be defined by the sum of its kinetic energy $\mathcal{K}(\boldsymbol{q}, \dot{\boldsymbol{q}})=\frac{1}{2} \dot{\boldsymbol{q}}^{T} M \dot{\boldsymbol{q}}$ and potential energy $\mathcal{U}(\boldsymbol{q})$ as

$$
E(\boldsymbol{q}, \dot{\boldsymbol{q}})=\frac{1}{2} \dot{\boldsymbol{q}}^{T} M \dot{\boldsymbol{q}}+\mathcal{U}(\boldsymbol{q}) .
$$

So, the time derivative of A.2 , i.e., the power, is given by

$$
\dot{E}(\boldsymbol{q}, \dot{\boldsymbol{q}})=\frac{1}{2} \dot{\boldsymbol{q}}^{T} \dot{M} \dot{\boldsymbol{q}}+\dot{\boldsymbol{q}}^{T} M \ddot{\boldsymbol{q}}+\frac{\partial \mathcal{U}(\boldsymbol{q})^{T}}{\partial \boldsymbol{q}} \dot{\boldsymbol{q}}
$$

Then, by substituting the system A.1 into A.3 we get

$$
\begin{aligned}
\dot{E}(\boldsymbol{q}, \dot{\boldsymbol{q}}) & =\frac{1}{2} \dot{\boldsymbol{q}}^{T} \dot{M} \dot{\boldsymbol{q}}+\dot{\boldsymbol{q}}^{T}(-C(\boldsymbol{q}, \dot{\boldsymbol{q}}) \dot{\boldsymbol{q}}-\boldsymbol{g}(\boldsymbol{q})+\boldsymbol{\tau})+{\frac{\partial \mathcal{U}(\boldsymbol{q})^{T}}{\partial \boldsymbol{q}} \dot{\boldsymbol{q}}} \\
& =\dot{\boldsymbol{q}}^{T}\left[\frac{1}{2} \dot{M} \dot{\boldsymbol{q}}-C(\boldsymbol{q}, \dot{\boldsymbol{q}})\right] \dot{\boldsymbol{q}}-\dot{\boldsymbol{q}}^{T} \frac{\partial \mathcal{U}(\boldsymbol{q})}{\partial \boldsymbol{q}}+\dot{\boldsymbol{q}}^{T} \boldsymbol{\tau}+\frac{\partial \mathcal{U}(\boldsymbol{q})^{T}}{\partial \boldsymbol{q}} \dot{\boldsymbol{q}} .(\mathrm{A} .4)
\end{aligned}
$$

By applying the skew-symmetry property [16] the first term of the right hand side of A.4 is null, then it remains

$$
\dot{E}(\boldsymbol{q}, \dot{\boldsymbol{q}})=\dot{\boldsymbol{q}}^{T} \boldsymbol{\tau}
$$


${ }_{540}$ If we use the generalized coordinate $\boldsymbol{\theta}$ instead of $\boldsymbol{q}$ and their corresponding torques $\overline{\boldsymbol{\tau}}$ instead of $\boldsymbol{\tau}$, we will get the same structure, as we will see next: the energy of the mechanical system (A.1) can be defined by the sum of its kinetic energy $\mathcal{K}(\boldsymbol{\theta}, \dot{\boldsymbol{\theta}})=\frac{1}{2} \dot{\boldsymbol{\theta}}^{T} A^{T} M A \dot{\boldsymbol{\theta}}$ and potential energy $\mathcal{U}(\boldsymbol{\theta})$ as

$$
E(\boldsymbol{\theta}, \dot{\boldsymbol{\theta}})=\frac{1}{2} \dot{\boldsymbol{\theta}}^{T} A^{T} M A \dot{\boldsymbol{\theta}}+\mathcal{U}(A \boldsymbol{\theta}+\boldsymbol{b})
$$

where by using equivalence 19 in 4.6 we get

$$
E(\boldsymbol{\theta}, \dot{\boldsymbol{\theta}})=\frac{1}{2} \dot{\boldsymbol{\theta}}^{T} \bar{M} \dot{\boldsymbol{\theta}}+\overline{\mathcal{U}}(\boldsymbol{\theta}) .
$$

${ }_{545}$ So, the time derivative of A.7), i.e. the power, is given by

$$
\dot{E}(\boldsymbol{\theta}, \dot{\boldsymbol{\theta}})=\frac{1}{2} \dot{\boldsymbol{\theta}}^{T} \dot{\bar{M}} \dot{\boldsymbol{\theta}}+\dot{\boldsymbol{\theta}}^{T} \bar{M} \ddot{\boldsymbol{\theta}}+\frac{\partial \overline{\mathcal{U}}(\boldsymbol{\theta})^{T}}{\partial \boldsymbol{\theta}} \dot{\boldsymbol{\theta}}
$$

By substituting the system (18) into A.8 we get

$$
\begin{aligned}
\dot{E}(\boldsymbol{\theta}, \dot{\boldsymbol{\theta}}) & =\frac{1}{2} \dot{\boldsymbol{\theta}}^{T} \dot{\bar{M}} \dot{\boldsymbol{\theta}}+\dot{\boldsymbol{\theta}}^{T}(-\bar{C}(\boldsymbol{\theta}, \dot{\boldsymbol{\theta}}) \dot{\boldsymbol{\theta}}-\overline{\boldsymbol{g}}(\boldsymbol{\theta})+\overline{\boldsymbol{\tau}})+{\frac{\partial \overline{\mathcal{U}}(\boldsymbol{\theta})^{T}}{\partial \boldsymbol{\theta}}}^{\dot{\boldsymbol{\theta}}} \\
& =\dot{\boldsymbol{\theta}}^{T}\left[\frac{1}{2} \dot{\bar{M}} \dot{\boldsymbol{\theta}}-\bar{C}(\boldsymbol{\theta}, \dot{\boldsymbol{\theta}})\right] \dot{\boldsymbol{\theta}}-\dot{\boldsymbol{\theta}}^{T} \frac{\partial \overline{\mathcal{U}}(\boldsymbol{\theta})}{\partial \boldsymbol{\theta}}+\dot{\boldsymbol{\theta}}^{T} \boldsymbol{\tau}+\frac{\partial \overline{\mathcal{U}}(\boldsymbol{\theta})^{T}}{\partial \boldsymbol{\theta}} \dot{\boldsymbol{\theta}},(\mathrm{A} .9)
\end{aligned}
$$

where $\overline{\boldsymbol{g}}(\boldsymbol{\theta})=\frac{\partial \overline{\mathcal{U}}(\boldsymbol{\theta})}{\partial \boldsymbol{\theta}}$. Thus, by applying the skew-symmetry property [16] the first term of the right hand side of $(A .9)$ vanishes. Therefore,

$$
\dot{E}(\boldsymbol{\theta}, \dot{\boldsymbol{\theta}})=\dot{\boldsymbol{\theta}}^{T} \overline{\boldsymbol{\tau}}
$$

By using 23 into A.10 we have

$$
\dot{E}(\boldsymbol{\theta}, \dot{\boldsymbol{\theta}})=\dot{\boldsymbol{\theta}}^{T} A^{T} \boldsymbol{\tau}=[A \dot{\boldsymbol{\theta}}]^{T} \boldsymbol{\tau},
$$

where by using the equivalence $\dot{\boldsymbol{q}}=A \dot{\boldsymbol{\theta}}$ and comparing with A.5, it is clear that $\dot{E}(\boldsymbol{q}, \dot{\boldsymbol{q}})=\dot{E}(\boldsymbol{\theta}, \dot{\boldsymbol{\theta}})$. 